\title{
Die Diffusion von Innovationen über Zeit und Raum Ein Überblick zu Ansätzen der Diffusionsforschung und evolutionären Innovationsökonomie'
}

\section{Einleitung}

Bei der alten Frage „Wie kommt das Neue in die Welt?“ steht ja nicht allein die Erschaffung des (vermeintlich) Neuen, sondern wie das Wissen darum (oder des damit verbundenen Artefaktes: Technik, Methode, Baustil, Ackerbaupraxis ...) seinen Weg in die Welt über Zeit und Raum findet. Neben der wissenschaftlichen Beschäftigung der Historiker über Wege des Wissens ${ }^{2}$ stehen auch angewandte Forschungsarbeiten, z.B. der Bodendenkmalpflege, 3 vor der Frage, von wo „etwas“ kommt, wer von wem lernt, wer bekommt, überlässt oder erobert hat.

In der interdisziplinären Betrachtung durch das Exzellenzcluster Topoi können möglicherweise auch die Wirtschaftswissenschaften einen Beitrag leisten, indem sie ihre systematische Betrachtung von Neuerungen und deren Verbreitung in einer Population von Wirtschaftssubjekten im Zuge der Diffusions- und Innovationsforschung kurz vorstellen und - als Nicht-Historiker zugegebenermaßen dilettantisch und höflicherweise in Frageform - der Altertumsforschung und Archäologie bekannt machen. Dieses möchte der vorliegende Beitrag versuchen.

Hierzu sollen zunächst notwendige begriffliche Klärungen zum besseren Verständnis vorgenommen werden. Danach sollen die Ansätze der wirtschaftswissenschaftlichen Diffusionsforschung und ihrer grundlegenden Modelle unter Beibehaltung der neoklassischen Gleichgewichtshypothese zur Verbreitung von neuen Produkten und Techniken erklärt werden. Die Schilderung der Erkenntnisse der neueren Innovationsforschung, basierend auf Schumpeter und den Ansätzen der evolutionären Innovationsökonomie schließen sich an, da diese die Auflösung der dichotomen Betrachtung der an der neoklassischen Gleichgewichtshypothese orientierten Modelle anbieten und im Gegensatz dazu

I Der Beitrag wurde am 2. Dezember 2009 als Vortrag auf dem Workshop „Mobilität und Wissenstransfer in diachroner und interdisziplinärer Perspektive“ der Research Group A II des Exzellenzclusters TOPOI in Berlin gehalten. Einzelne Textteile und Abbildungen sind ohne besondere Kennzeichnung aus früheren Arbeiten des Autors entnommen (vor allem aus Dreher I997, insbesondere Kapitel 3; Dreher 2008).

2 Z.B. Renn et al. 2009 .

3 Z.B. Behrends et al. I996. 
Vielfalt, Akteure, Interaktionen und soziokulturelle Kontexte in den Vordergrund stellen. Die besagten Fragen und Anregungen schließen den Beitrag ab.

\section{„Innovation“ in den Wirtschaftswissenschaften}

Vom Wortstamm her bedeutet Innovation „Veränderung“, etwas Neues zu schaffen. Für die Wirtschaftswissenschaften ist sicherlich Schumpeter zwischenzeitlich derjenige, der am häufigsten für diesen Begriff bemüht wird („The fundamental input that sets and keeps the capitalist engine in motion comes from the new consumer goods, the new methods of production or transportation, the new markets, the new forms of industry organisation that capitalist enterprises creates. “). Demnach ist ein innovativer Unternehmer derjenige, der durch die Realisierung einer neuen Idee (Produkt, Konzept, Organisation, Dienstleistung, Herstellverfahren, Werbemaßnahme, Farbe der Verpackung, neues Auslieferungsdatum) bestehende Zusammenhänge (zer)stört ${ }^{4}$ und - wenn er erfolgreich ist - Nachahmer findet und neue Wachstumsschübe in der Wirtschaft auslösen kann.

In der Zeit nach Schumpeter wurde aber der Innovationsbegriff im Wesentlichen auf technische Neuerungen begrenzt. So beschreiben Freeman und Soete Innovationen als einen Prozess, der die Entwicklung, das Design, die Produktion und die Vermarktung eines neuen (oder verbesserten) Produktes oder den erstmaligen Einsatz eines neuen (oder verbesserten) Produktionsverfahrens umfasst. 5 Aus dieser rudimentären Darstellung ${ }^{6}$ wird eines deutlich: Innovation ist ohne Bezugssystem nicht zu verstehen. Für das Bezugssystem sind folgende Aspekte zu präzisieren:

- Zeithorizont: Innovation ist nur im Zeitverlauf zu verstehen. Etwas ist anders, neu, verändert im Vergleich zu einem früheren Zeitpunkt;

- Gegenstand: Vielfach wird unter Innovation ein forschungs- und entwicklungsgetriebenes neues Produkt oder ein neues Verfahren verstanden, aber auch andere Aspekte geraten zunehmend in das Blickfeld (z.B. Dienstleistungen);

- das eingegangene Risiko;

- $\quad$ der (subjektive) Neuigkeitsgrad: Was für den einen bahnbrechend zu sein scheint, empfindet ein anderer als alten Hut;

- die Betrachtungsebene: Eine Innovation kann etwa für den einzelnen Arbeitnehmer, für den Betrieb, für den Markt eines Unternehmens oder gar gesamtwirtschaftlich neu sein.

Für diesen Beitrag wird der Begriff Innovation auf betriebliche Neuerungen und Veränderungen in Unternehmen oder Organisationen beschränkt. Entwicklungen in der Ge-

\footnotetext{
4 Schumpeter selbst spricht von kreativer Zerstörung (Schumpeter I942).

5 Freeman/Soete I997.

6 Mehr in Grupp I997; Dreher I997.
} 
sellschaft oder Veränderungen durch den Einzelnen bleiben unberücksichtigt. Erfolgreich ist eine Innovation bei wirtschaftlichem Erfolg, d.h. beispielsweise mehr Umsätze, höhere Marktanteile, weniger Kosten, mehr Beschäftigung. Andere Erfolgsdimensionen, insbesondere kulturelle oder ästhetische Beiträge, bleiben ausgeklammert. Das Innovationssystem beschreibt den Rahmen, in dem sich die Betriebe und betrieblichen Akteure bewegen (können), aus dem sie ihre materiellen oder immateriellen Ressourcen für betriebliche Innovationen beziehen und in dem (Inter-)Aktionen anderer als Signale zu empfangen und zu verarbeiten sind. 7

\section{Die Forschung zur Diffusion von Innovationen}

\section{Die Klassiker}

Die Forschung über die Diffusion von Innovationen hat historisch ihren Ursprung in der Betrachtung von kulturellen und agrarsoziologischen Verhaltensweisen und ihrer räumlichen Ausbreitung im Zeitverlauf. Neben der epidemiologischen Forschung in der Medizin folgten Überlegungen zur Verbreitung von Investitions- und Konsumgütern.

Die Motivation, sich mit der Verbreitung von Innovationen zu befassen, erwächst aus der Beobachtung, dass sich Innovationen nicht spontan, sondern über die Zeit verteilt im Raum verbreiten und dass diese Verbreitungsprozesse erst bei einer Sättigungsgrenze aufhören und nicht vorher abbrechen. ${ }^{8}$ Hinzu kommt, dass die Verbreitung von Innovationen - zumindest aus Sicht der noch nicht sehr ,alten“ Wirtschaftswissenschaften - ein ,zäher‘, langwieriger, oft Jahrzehnte dauernder Vorgang ist und die Adoptionsrate bei unterschiedlichen Innovationen in verschiedenen Sektoren oder gesellschaftlichen Kontexten erheblich variieren kann.9 Ihre Relevanz für die Wirtschaftswissenschaft zieht die Diffusionsforschung daraus, dass die Profite, die sich Volkswirtschaft, Sektoren oder Unternehmen von Innovationen erhoffen, erst durch die Realisierung und Ausbreitung dieser Innovationen entstehen ${ }^{\mathrm{IO}}$ und damit Prozesse und Hindernisse der Verbreitung von Innovationen $\mathrm{zu}$ erforschen sind.

Die verschiedenen Stränge der Diffusionsforschung wurden von Rogers in seinem Standardwerk zusammengefasst und verallgemeinert. Demnach ist Diffusion als der Prozess definiert, bei dem Informationen über Innovationen durch bestimmte Kommunikationskanäle über die Zeit unter den Mitgliedern eines sozialen Systems kommuniziert wer-

7 Gängige Definitionen von Innovationssystemen beschränken sich auf das Hervorbringen von Technologien,

z. B. Metcalfe I995.

8 Dosi i99I, I85.

9 Rosenberg I976, I9I; empirisch argumentiert Ray I989, I6.

I0 Rosenberg I982, I9. 
den. ${ }^{\text {II }}$ Das typische Diffusionsmuster wird weiter durch die mögliche Heterogenität der Mitglieder in einem sozialen System hinsichtlich ihrer Haltung zu und ihren Erwartungen über den Nutzen einer Innovation bestimmt. Da sie aber nicht isoliert im sozialen System existieren, tauschen sie ihre Erfahrungen aus und lernen gegebenenfalls durch Imitation der Verhaltensweisen anderer. Laut Rogers gehen alle theoretischen und empirischen Diffusionsstudien somit davon aus, dass Innovationen nicht spontan und unmittelbar komplett in ein soziales System diffundieren. ${ }^{\text {I2 }}$ Die S-förmige Diffusionskurve scheint deshalb ein Grundphänomen zu sein.

Rogers entwickelte einen vierstufigen Prozess der Diffusion von der Übermittlung der Information als den ersten Schritt bis zur Adoptionsentscheidung. ${ }^{13}$ Die vier Phasen sind die Wissensübermittlung, das Gewinnen einer Überzeugung, die Entscheidung und Implementation sowie eine Bestätigung der Richtigkeit der Entscheidung. Die in die Entscheidung einfließenden Eigenschaften der jeweiligen Innovation sind:

- Die relative Vorteilhaftigkeit der Innovation,

- die Kompatibilität hinsichtlich früherer Erfahrungen, existierender Werte sowie individueller Bedürfnisse und Erwartungen,

- die Komplexität hinsichtlich des Aufwandes, die Innovation zu verstehen und anwenden zu können,

- die Probierbarkeit, die es erlaubt, in unterschiedlichem Maße mit einer Innovation vorher zu experimentieren, bevor man eine endgültige Entscheidung trifft sowie

- die Beobachtbarkeit der Ergebnisse der Anwendung der Innovation für den Entscheidenden wie auch für die anderen Mitglieder des sozialen Systems.

Alle diese Variablen sind, außer der Komplexität, mit der Adoptionsrate einer Innovation positiv korreliert.

Da das Potential an Anwendern in seinem Entscheidungsverhalten zur Anwendung einer Innovation nicht notwendigerweise homogen ${ }^{\text {I4 }}$ ist, hat Rogers die Adopter hinsichtlich ihres Entscheidungszeitpunktes kategorisiert, indem er die Standardabweichung vom durchschnittlichen Adoptionszeitpunkt als Maßstab verwendet. Brown, Rogers sowie andere präsentieren empirische Belege, die die Normalverteilung der Adopter über die Zeit plausibel machen. ${ }^{15}$ Kumuliert man die Zahlen der Adopter, erhält man eine symmetrische S-förmige Kurve, die mathematisch durch eine Gompertz-Funktion beschrieben werden kann.

In seinem Beitrag versucht Edwin Mansfield die grundlegenden Erfahrungen in ein ökonomisches Modell zu übertragen, das die Adoptionsraten industrieller Innovationen

II Rogers I983, 5 .

I2 Rogers I983.

I3 Rogers I983.

I4 Ursachen sind individuelle Merkmale des Adopters, soziale, ökonomische Kriterien, Kommunikationsverhalten, Innovationsfreude, Erfahrungen. Bedürfnisse.

I5 Brown I98I; Rogers I983. 
erklärt, und testet sein Modell für eine Anzahl von industriellen Prozessinnovationen in unterschiedlichen Sektoren. ${ }^{16}$ Anders als Rogers, ausgehend von einem homogenen Umfeld für die Anwender der Innovation und einem über den Diffusionsprozess konstanten Adopterpotential, verwendet Mansfield zur Beschreibung eine logistische Verbreitungsfunktion. Diese logistische Funktion enthält eine Imitationsrate, die neben einer industriesektorspezifischen Konstanten wiederum abhängig ist von der Profitabilität der Innovation und dem Umfang der vorzunehmenden Investition. Die Kurve nimmt einen S-förmigen Verlauf und wird durch die Gesamtzahl der möglichen Adopter und der Lage des Wendepunktes $t_{0}$ bestimmt. Im von Mansfield beschriebenen symmetrischen Fall liegt dieser bei der Hälfte des Adopterpotentials (Abb. I).

Die grundlegenden Schlussfolgerungen, die sich aus der Anwendung dieses Modells ergeben, lassen sich folgendermaßen zusammenfassen: ${ }^{17}$

- Die Diffusion von Innovationen ist grundsätzlich ein Lernprozess, der anhand eines so genannten Bandwaggon-Effekts illustriert wird; einem Effekt, der die Infizierung von Mitgliedern eines sozialen Systems, hier Firmen, durch Wissen voraussetzt und die imitierenden Firmen den Innovatoren nachfolgen lässt.

- $\quad$ Es gibt eine Analogie zu den klassischen Lernmodellen und psychologischen Gesetzen, ${ }^{18}$ in denen der Umfang des Lernerfolges eine Funktion vorher akkumulierten Wissens ist. In der ökonomischen Anwendung lernen Firmen von den Erfahrungen anderer, die bereits die Innovation anwenden.

- Die Reaktionszeit und die Lernrate hängen von der Intensität des Stimulus ab, den die Innovation ausübt. Dieser Stimulus wird durch die Profitabilität der Prozessinnovation dargestellt. Er hängt wiederum von folgenden Faktoren ab:

- Dem Umfang des ökonomischen Vorteils der Innovation gegenüber älteren Methoden oder Produkten,

- dem Umfang der Unsicherheit, der mit der Nutzung der Innovation und dem Ersteinsatz verbunden ist,

- dem Umfang des eigenen Aufwandes des potentiellen Adopters, der notwendig ist, um die Innovation auszuprobieren und

- dem Umfang der Reduktion der ursprünglichen Unsicherheit über die Leistung der Innovation nach der Adoptionsentscheidung. ${ }^{\text {I9 }}$

In weiterführenden Untersuchungen ergänzt Mansfield noch die im ursprünglichen Basismodell nicht näher spezifizierten Determinanten: ${ }^{20}$

i6 Mansfield i96I.

I7 Vgl. dazu Mansfield I968a; I968b; I984; Mansfield et al. I977 sowie weitere empirische Ergebnisse, die dieses Basismodell als Beschreibungsinstrument für empirische Phänomene und für die ökonomische Interpretation stützen, insbesondere: Blackman I97I; Hsia I973; Simon I975 und Romeo I977.

I8 Vgl. Rogers i983.

I9 Mansfield ig68b.

20 Mansfield i984. 


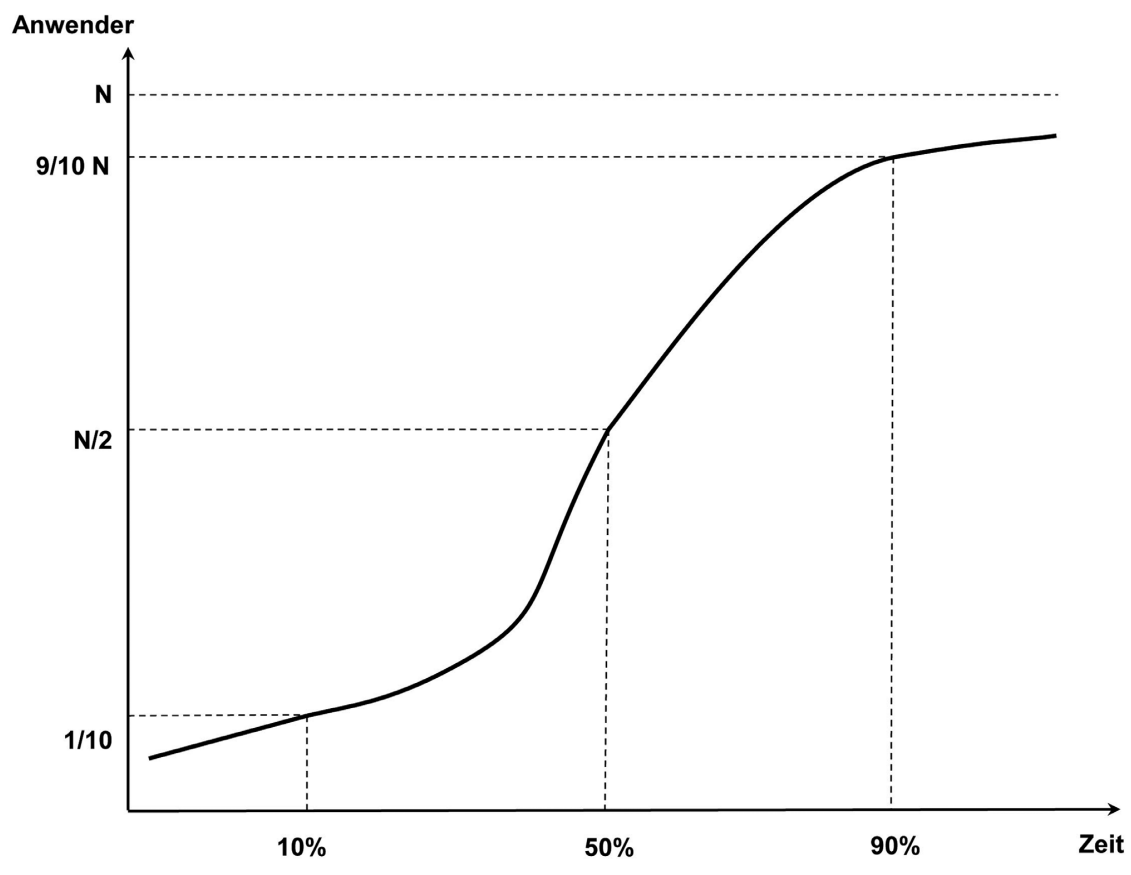

Abb. 1 | Symmetrisch-logistische Diffusionskurve (aus Dreher I997, 57).

- Die wissenschaftlichen Fähigkeiten und der Ausbildungsstand des jeweiligen industriellen Sektors beeinflussen die Adoptionsentscheidung der Unternehmen,

- Wettbewerbsintensität in einem Sektor erhöht die Wahrscheinlichkeit der Adoption und

- Erfahrungen, die außerhalb des betreffenden industriellen Sektors mit einer Innovation gewonnen werden, können die Unsicherheit über die Effekte der Innovationen reduzieren.

Diese von Mansfield ermittelten Determinanten und Schlussfolgerungen bilden das Rückgrat für die weitere Modellierung der Verbreitung von Innovationen in Unternehmen.

Weiterentwicklungen des Mansfield-Modells

Die Weiterentwicklung der ökonomischen Modelle der Diffusion von Innovationen richtete sich anhand der Kritik an dem Basismodell von Mansfield aus. Grübler strukturiert diese Diskussion und damit die Weiterentwicklung in unterschiedliche Richtungen mittels vier Ansatzpunkten: ${ }^{2 I}$

2I Grübler 1992, I4; neuere Übersicht über Entwicklungslinien der Modelle bei Lissoni/Metcalfe I994; Karshenas/Stoneman I995, Geroski 2000, Frenzel/Grupp 2009. 
I. Mathematische Eigenschaften des Modells, insbesondere die symmetrische Eigenschaft der logistischen Kurve und die fehlende Herleitung der zugrunde gelegten Normalverteilung auf der Basis eines ökonomischen Zusammenhanges,

2. die dichotomische Einteilung in Adopter und Nichtadopter einer Innovation als unrealistische Annahme,

3. die Konzentration der Modellentwicklung auf Prozessinnovationen, die zu einem zweiten Strang an Modellen für langlebige Konsumgüter zu Marketingzwecken geführt hat sowie

4. die Einengung auf $\mathrm{zu}$ wenige, die Adoptionsentscheidung beeinflussenden Variablen, ${ }^{22}$ das Nichtberücksichtigen möglicher anderer Faktoren, die die Diffusion beeinflussen, sowie das von Mansfield implizit unterstellte statische Verhalten von Variablen und Randbedingungen.

Ad r.: Die logistische S-Kurve wurde von Mansfield aus einem anderen Kontext in die Ökonomie übertragen. Das Verbreiten von Informationen, die Verbreitung einer ansteckenden Krankheit wie auch Theorien des sozialen Lernens bildeten die Grundlagen, die in seinem Modell zu der Verwendung der symmetrischen logistischen S-Kurve führten. Die Weiterentwicklungen halten diese Übertragung für beschränkend. ${ }^{23}$ Im Einzelnen führten folgende Kritikpunkte zur Weiterentwicklung:

- $\quad$ Die aus der logistischen S-Kurve resultierende Symmetrie am Wendepunkt $t_{\circ}$ bei N/2 zeigt, dass die Wahrscheinlichkeit der Adoption über die gesamte Zeit konstant bleibt und damit die Interaktion von Adoptern und Nichtadoptern sich im Zeitverlauf ebenfalls nicht ändert.

- Der logistischen Kurve liegt die Annahme einer homogenen Population dergestalt zugrunde, dass potentielle Adopter dieselben Kalküle über den gesamten Diffusionszeitraum anwenden.

Diese angenommene „mangelnde mathematische Flexibilität“ führte zur Weiterentwicklung in der mathematischen Darstellung der Funktionen von Verbreitungsprozessen. Diese Weiterentwicklungen sind modifizierte Exponentialfunktionen, ${ }^{24}$ z. B. in der Tradition des Bass-Modells, die Floyd-Kurve, die Gompertz-Funktion und kumulierte normale oder lognormale Verbreitungsmuster. ${ }^{25}$ Andere versuchten durch Ergänzung von Parametern die logistische Funktion $\mathrm{zu}$ variabilisieren. ${ }^{26}$

22 Mansfield I96r ließ für die Adoptionsentscheidung nur Sektorspezifika, angenommene Profitabilität der Innovation und Umfang der zur Realisierung der Innovation notwendigen Investition zu.

23 Oder deutlicher: „One may also argue, specifically with respect to the Mansfield model, that there is no good reason in that model for expecting the diffusion curve to be logistic. The logistic results purely from the arbitrary restrictions placed on the Taylor's series expansion, Stoneman I983“, zitiert nach Karsheas/Stoneman I995, 272. 24 Eine gute Übersicht geben Mahajan et al. I99I in ihrer Tabelle I44-I45.

25 Vgl. Bass I969; Davis I979; Floyd I962; Hendry I972; Dixon I980; Jeuland I981; Nelder I962; McGowan I986; von Bertalanffy i957; Richards I959.

26 Sharif/Kabir I976; Easingwood et al. I981; Easingwood et al I983; Teotia/Raju I986; Bewley/Fiebig I988. 
Ein weiteres formales Problem ist die Herleitung bzw. Begründung der logistischen Kurve aus einer zugrunde gelegten Normalverteilung heraus. Mansfield übernahm die Erfahrungen aus der epidemiologischen Forschung, um aus der Verteilung von potentiellen Adoptern die logistische Kurve herzuleiten. ${ }^{27}$ In der Tat wurde damals die Übertragung nicht auf ökonomisch begründbare Verteilungen zurückgeführt, deren Kumulierung ebenfalls ähnlich geartete Kurven ergab. In der Folge konnte nachgewiesen werden, dass sich die (weiter-)entwickelten Funktionen zur Beschreibung von Diffusionsverläufen von ökonomisch begründbaren Verteilungen wichtiger Einflussgrößen ableiten lassen, z.B. aus der Verteilung der Firmengrößen, ${ }^{28}$ der Einkommensverteilung ${ }^{29}$ oder der Wahrscheinlichkeit der unterschiedlichen Nutzenfunktionen potentieller Adopter. ${ }^{\circ}$

Ad 2.: Die noch von Rogers formulierte Einteilung einer Population potentieller Adopter in Anwender und in Nichtanwender ist für die ökonomische Anwendung eine zu starke Vereinfachung..$^{3 \mathrm{D}}$ Die ökonomischen Diffusionsmodelle in der Nachfolge Mansfields gehen in der Regel von einer Anwendung pro Adopter aus. Dabei treten folgende drei Probleme auf:

- Die Möglichkeit, dass ein Adopter eine Innovation zwar zunächst akzeptiert, aber durch Implementationsschwierigkeiten einen Misserfolg erfährt und es gegebenenfalls später noch einmal versucht, wird nicht berücksichtigt (Re-Infektion).

- $\quad$ Ein Adopter, z. B. eine größere Firma, kann bestimmte Innovationen mehrfach in Anwendung bringen (z.B. mehrere Maschinen). Dies erklärt empirisch auftauchende Differenzen zwischen Verkaufszahlen von Herstellern und Untersuchungen zu Anwenderquoten.

- Die Tatsache, dass eine Innovation nicht in ein Vakuum diffundiert, sondern alte Verfahrensweisen oder Produkte ersetzt oder verdrängt und daher die Diffusion vielfach ein Substitutionsphänomen ist, erfordert neue Modellüberlegungen.

Auf der Basis von Analogien aus der Biologie wird eine Wettbewerbssituation zwischen zwei Techniken oder technisch-organisatorischen Konzepten angenommen, die um ihren relativen Anteil an der Gesamtpopulation kämpfen. In der Anwendung für die Wirtschaftswissenschaft wurde erstmals durch Fisher, Pry und Blackman so ein Modell zum technischen Wandel entwickelt. ${ }^{2}$ Fisher und Pry gehen von den Annahmen aus, dass

I. der Substitutionsprozess unter Wettbewerbsbedingungen verläuft,

2. die Substitution fortschreitet bis zu einem kompletten Take-over der einen Technik, insofern es ihr gelingt, sich mit einem kleineren Prozentsatz in die Gesamtpopulation einzuklinken (Lock-in) sowie

27 Mansfield I96I.

28 Davies I979.

29 Russell i980.

30 Gottinger 1986

3I Rogers I983.

32 Fisher/Pry I97I; Blackman I97I. 
3. der Anteil der einen Technik proportional zu dem verbleibenden Anteil der anderen Technik ist.

Damit ähnelt das Fisher/Pry-Modell in der grafischen Darstellung dem logistischen Modell. Der Vorteil hierbei liegt allerdings darin, dass die jeweiligen Anteile der konkurrierenden Innovationen nicht nur anhand der absoluten Anzahl von Firmen oder verwendeten technischen Artefakten berechenbar sind, sondern auch hinsichtlich der Anteile an Produktionskapazitäten, Output etc., bestimmt werden können. Das Modell wurde durch Blackman bei sektoriellen Studien angewendet.

Der Hauptnachteil des Fisher/Pry-Modells ist die Tatsache, dass es nur mit zwei Wettbewerbern, d.h. zwei Technologien oder anderen Innovationen funktioniert. Eine Erweiterung des Fisher/Pry-Modells von einer einfachen Substitution zu einer multiplen Substitution wurde zuerst von Marchetti und Nakicenovic vorgeschlagen.33 Nakicenovic hat ein solches Modell für die Analyse der Weltenergiebilanz hinsichtlich der Anteile unterschiedlicher Primär-Energieträger verwendet.34 Für Produktinnovationen wurden vergleichbare Überlegungen durch Norton und Bass ${ }^{35}$ durchgeführt. ${ }^{6} 6$

Ad 3.: Die Konzentration der Diffusionsmodelle auf Prozessinnovationen hat Marketingforscher veranlasst, eigenständige Modelle für die Verbreitung von langlebigen Konsumgütern zu entwickeln. Ausgangspunkte der Überlegungen waren für den Diffusionsprozess wichtige Unterschiede zwischen Prozess- und Produktinnovationen:

- Die Entscheidungskalküle der Adopter unterscheiden sich bei Prozess- und Produktinnovationen. Bei Prozessinnovationen handelt es sich in der Regel um Investitionsentscheidungen, die von Firmen getroffen werden. Bei den Entscheidungsprozessen von Firmen spielt die Profitabilität der Prozessinnovation die dominierende Rolle und der Entscheidungsprozess scheint vergleichsweise rational zu sein. Bei Konsumgütern spielen andere Aspekte, z.B. individueller Nutzen, Prestige, Preis/Einkommensrelation etc. eher eine Rolle, die einzelne Konsumenten zum Kauf eines Gutes bewegen.

- Prozessinnovationen sind oft inkrementale Verbesserungen bestehender Verfahren, wie sie sich beispielsweise in verbesserten Maschinen oder Vorgehensweisen äußern. Im Gegensatz zu Konsumgütern ist die Verbreitung vielfach nicht durch Einflussnahme auf Marktstrukturen, wie beispielsweise durch Schutzrechte oder Patente, beeinträchtigt. Im Konsumgüterbereich können Patente oder andere Schutzrechte zeitweise zu monopolistischen Strukturen führen, die erst sehr viel später durch oligopolitische Strukturen auf Anbieterseite abgelöst werden können. Daraus ergeben sich für anbietende Unternehmen unterschiedliche Strategiemuster.

33 Marchetti/Nakicenovic I979.

34 Nakicenovic I984; ähnlich auch Habermeier I989.

35 Norton/Bass i987.

36 Fundamentale Kritik durch Batten I982; Bhargava I989 (erwähnt bei Bruckner et al. I993, die einen evolutionären Ansatz unternehmen). 
Basismodell für die marketing-orientierten Diffusionsmodelle ist der Ansatz, der von Bass entwickelt wurde. 37 Er entwickelte ein Diffusionsmodell, das den Erstkauf langlebiger Konsumgüter beschreibt. Er geht dabei von zwei Faktoren aus:

- Von dem Koeffizienten der Innovation, der in Beziehung zur Anzahl der (Erst)Innovatoren steht, die das Produkt kaufen und

- $\quad$ von dem Imitationskoeffizienten, der in Beziehung zu dem Rest der Gesamtpopulation (sog. Imitatoren) steht und sich in seinem Verhalten durch die Imitation der Innovatoren und die Verwendung von deren Erfahrungen auszeichnet.

Die notwendigen Kommunikationskanäle zur Erschließung der Innovatoren und der Imitatoren sind einmal externe Kanäle wie der Einsatz von Massenmedien, Werbung usw., die vor allem die Innovatoren ansprechen sollen. Der interne Kommunikationskanal läuft über interpersonale Kommunikation und sorgt dafür, dass die Imitatoren von den Innovatoren lernen.

Obwohl sich die Rollen der beeinflussenden Faktoren der Adopterentscheidungen zwischen den marketing-orientierten Modellen ${ }^{38}$ und den allgemeinen Diffusionsmodellen tendenziell unterscheiden, ist die formal-analytische Beschreibung beider Ansätze ähnlich39 und es können die gleichen Schlussfolgerungen hinsichtlich der Verläufe der Diffusionsprozesse von Innovationen gezogen werden. $4^{\circ}$

\section{Die Aufgabe der Homogenitätsannahme für die Adopterpopulation}

Die vierte kritische Hinterfragung hatte die Schöpfung einer neuen Modellfamilie zur Folge: In den grundlegenden Arbeiten von Mansfield ist die Verbreitung der Information über die Vorteilhaftigkeit einer Innovation diejenige Variable, die für den S-förmigen Verlauf der Diffusionskurve verantwortlich ist. Sie wirkt nämlich bei steigender Verbreitung minimierend auf die Unsicherheit potentieller Adopter. ${ }^{4}$ Die Steilheit der Diffusionskurve wird durch den zu erwartenden Profit aus der Innovation sowie aus dem Umfang der zur Realisierung der Innovation notwendigen Investition bestimmt. Diese beiden Determinanten wie auch die aus analytischen Gründen exogen vorgegebene Gesamtzahl potentieller Adopter und deren Homogenität bleiben über den gesamten Diffusionsverlauf konstant.

Gegen beide Grundüberlegungen wurde massiv Kritik ${ }^{42}$ geübt, die zur Entwicklung neuer Modellansätze führte. Die Kritikpunkte sind:

37 Bass i969.

38 Für einen Überblick vgl. Klophaus I995; Mahajan et al. I99I; Gierl I987, Geroski 2000; Frenzel/Grupp 2009 .

39 Vgl. Grübler 1992, 23.

40 Vgl. Grübler I992, 24; Lissoni/Metcalfe I994, II5.

$4 \mathrm{I}$ Stoneman I987, 5I nennt dies daher auch einen „,informationsbasierten“ Ansatz.

42 Ewers et al. I990, 25ff.; Maas I990, 37ff.; Erdmann I993. II2. 
- $\quad$ Die Lernprozesse sind gerade bei Prozessinnovationen sehr viel komplexer, als dass sie einfach durch ,Infektion ' dargestellt werden können. ${ }^{43}$ Fähigkeiten, Attitüden, Präferenzen, organisatorische Strukturen, Lernerfahrungen in benachbarten Innovationsbereichen etc. beeinflussen das Lernverhalten derart, dass beispielsweise trotz des Wissens über die Innovation die Adoptionsentscheidung zurückgestellt wird. Die Annahmen der Homogenität der Adopter und des konstant bleibenden Adopterpotentials werden daher verworfen.

- Die Annahme, dass die Profitabilität der Innovation und der für sie notwendige Aufwand über die Zeit konstant bleibt, würde anderen Marktgesetzen widersprechen:

- So bleibt der Preis beim Wettbewerb unter Anbietern über die Zeit nicht konstant, sondern wird bei steigendem Absatz und möglichen Skaleneffekten sinken und damit für bisher nicht infrage kommende Anwendungsbereiche profitabel werden;44

- bei sinkenden Preisen ändert sich ebenfalls der Umfang der zur Realisierung der Innovation notwendigen Investition und verändert damit das Kalkül des vor der Adoptionsentscheidung stehenden Unternehmens.

Aus dieser Kritik heraus entwickelte sich eine Anzahl von Modellen, die sich unter dem Begriff „Probit-Ansätze“ zusammenfassen lassen. Probit-Ansätze unterstellen einen Diffusionsverlauf, der sich auf die individuellen Adoptionsentscheidungen und die Heterogenität der Adopter zurückführen lässt.45 Die potentiellen Adoptoren reagieren auf das angebotene Potential von Innovationen erst, wenn diese einen für sie kritischen Wert überschreiten. Da solche Reaktionen trotz vollständiger Information (d.h. alle Marktteilnehmer wissen sofort alles) aufgrund der Heterogenität nicht gleichzeitig bei allen Adoptoren auftreten, muss der zur Adoptionsentscheidung erforderliche kritische Wert nicht für alle potentiellen Adoptoren identisch und/oder aufgrund exogener oder endogener Faktoren im Zeitverlauf konstant sein.

Der Probit-Ansatz erlaubt eine Modellbildung, die diese Variabilisierung über die Zeit sowie die Hinzufügung unterschiedlichster erklärender Variablen vornimmt. Im Gegensatz zur epidemiologischen Vorstellung befinden sich die Akteure über den gesamten Modellverlauf in einem Gleichgewicht des Technikeinsatzes, das ökonomisch-rationalen Kalkülen der Unternehmen entspricht. ${ }^{6}$ In der Literatur findet sich ein breites Spektrum von Modellen, die verschiedene Thesen und als relevant angesehene Variablen wie Charakteristika der Innovation, Merkmale des Unternehmens und Verhaltensweisen der Entscheider testen. 47

43 Ewers et al. I990, 27.

44 Ewers et al. I990, 26.

45 Stoneman I983, 97; Thiel I988, 108.

46 Ewers et al. I990, 28. Sie werden auch Equilibrum-Ansätze genannt; vgl. Lissoni/Metcalfe I994.

47 Vgl. Übersicht, z. B. bei Duchesneau et al. I979; Kleine I983; Maas I990. 
Die vorgegebene Gesamtpopulation potentieller Adoptoren muss ebenfalls nicht konstant sein. Aber die Argumentation zur „Sättigungsgrenze“ bei Ewers, Becker, Fritsch ${ }^{8}$ wirkt nicht überzeugend: Mansfield definierte zwar keine endogene, aus dem Diffusionsverlauf sich ergebende Sättigungsgrenze, sondern gab für empirische Analysen die sinnvolle Abgrenzung einer Gesamtpopulation vor. In dem Fall von einer „exogen vorgegebenen Sättigungsgrenze“ zu sprechen, wäre eine Überinterpretation. Relevanter sind da die Auswirkungen auf das Adopterpotential, die durch eine Veränderung der Innovation durch technischen Fortschritt im Laufe des Diffusionsprozesses hervorgerufen wird. Dabei sind drei Fälle zu unterscheiden:

- Technische Weiterentwicklung kann die Anzahl potentieller Adopter sprunghaft erhöhen (Beispiel Mikroelektronik). So kann technischer Fortschritt die Erfüllung neuer oder zusätzlicher Funktionen durch eine Innovation ermöglichen und damit neue Absatzpotentiale erschließen.49

- Die Veränderung der Innovation durch den technischen Fortschritt wie auch das Wechselspiel unterschiedlicher Variablen, das die relative Profitabilität der Innovation sowie den Umfang der notwendigen Investition verändert, hat Einfluss auf die Nachfrage und das Wachstum des Marktes der Innovation. Metcalfe problematisierte und formulierte diesen Interaktionsaspekt zwischen Nachfrage und Angebot. ${ }^{\circ}$

- Bei Innovationen im Wettbewerb können zwei Entwicklungen eintreten:

- der so genannte Segelschiff-Effekt ${ }^{5 \mathrm{I}}$ weist auf die technischen Verbesserungen hin, die eine alte Technologie erfahren kann, wenn sie durch eine neue herausgefordert wird, wie dies bei den technologischen Sprüngen der Segeltechnik beim Auftauchen der Dampfschiffe der Fall war;

- Skiadas untersuchte den Fall, in dem die Diffusion einer Technik durch das Auftauchen einer anderen, neuen Technik plötzlich abbrach..$^{22}$

Integration in der empirischen Forschung

Folgt man den beiden Modellfamilien (Mansfield und Weiterentwicklungen versus ProbitModelle) muss man für die empirische Analyse zwei Gruppen von zu untersuchenden Determinanten unterscheiden:53

- Einflüsse der Innovation auf die Wirtschaftlichkeitsbetrachtung der Unternehmen sowie

48 Ewers et al. I990, 26.

49 Gold I98I, I983.

50 Metcalfe I983; vgl. auch Thirtle/Ruttan 1987; Kortmann I995.

5I Vgl. Rosenberg 1976.

52 Skiadas I986; er untersuchte die Diffusion am Beispiel von Verfahren zur Stahlerzeugung.

53 Vgl. Maas I990, 54 . 
- unternehmensbezogene Merkmale, insbesondere Charakteristika der am Innovationsprozess Beteiligten und betriebliche Merkmale des potentiellen Adopters (Informationsverhalten, Organisationsstruktur, Strategie- und Finanzierungsverhalten).

Während die erste Gruppe von Determinanten den von Mansfield postulierten Variablen Profitabilität der Innovation, Komplexität, Realisierungsaufwand etc. entspricht, untersucht die andere Determinantengruppe Aspekte, die die Heterogenität der potentiellen Adoptoren unterstreichen und folgt damit den Modellvorstellungen der Probit-Ansätze. ${ }^{54}$

Die Dichotomie der theoretischen Ansätze lässt sich bei Durchsicht der empirischen Befunde55 nur schwer aufrecht erhalten, zumal der S-förmige Verlauf der Diffusionskurve gemeinsam beobachtet wird. Gerade für eine realistische Ermittlung der Adopter und ihrer Potentiale kann aber eine Kombination der Determinanten hilfreich sein. Diese wären:

- Identifikation von Akteuren mit fehlenden Einsatzvoraussetzungen,

- betriebswirtschaftliche Kalküle, abhängig vom technischen Stand und der Kompatibilität mit Anwenderstrukturen in Produkt und Produktion,

- Verhaltensweisen der an Adoptionsentscheidungen beteiligten Personen sowie

- Informations- und Kommunikationsdefizite.

Im dynamischen Verhalten bleibt der Anteil der theoretisch möglichen Adopterpopulation, z. B. eine Branche oder ein Sektor, für die/ den die Einsatzvoraussetzungen fehlen, stabil. Die betriebswirtschaftlichen Kalküle verändern sich aber entsprechend der Darstellung von Gold, da sich die Innovation im Zeitverlauf verändert und zu besseren und passfähigeren Angeboten führt. ${ }^{6}$ Die Unsicherheiten, die durch qualifikatorische und organisatorische Veränderungen hervorgerufen werden, und die Informationsdefizite werden insgesamt mit der Zahl der Anwendungen im Zeitverlauf analog zu den Probit-Modellen sowie dem Mansfield-Modell abnehmen (Abb. 2).

Gerade die Vielfalt bei der empirischen Analyse und die Notwendigkeit, die gegensätzlichen Annahmengerüste der Modelle aufzulösen, legen es nahe, über Innovationen und dynamisches Wirtschaftsverhalten neu und ohne Gleichgewichtshypothese nachzudenken.

\section{Die evolutionäre Innovationsökonomie}

\section{J.A. Schumpeter - Der Klassiker}

Der Ausgangspunkt der Betrachtungen von Schumpeter war die „Wirtschaftliche Entwicklung" und die Erklärung von Konjunkturzyklen. ${ }^{57}$ Auf der Suche nach den Ursachen der Überwindung von statischen Situationen sind es die, innovativen Unternehmer', die mit

54 Maas I990; Ewers et al. I990; Kleine I983; Schwitalla I993.

55 Vgl. Dreher I997, Kapitel 3.

56 Gold i98I.

57 Vgl. Schumpeter I9II. 


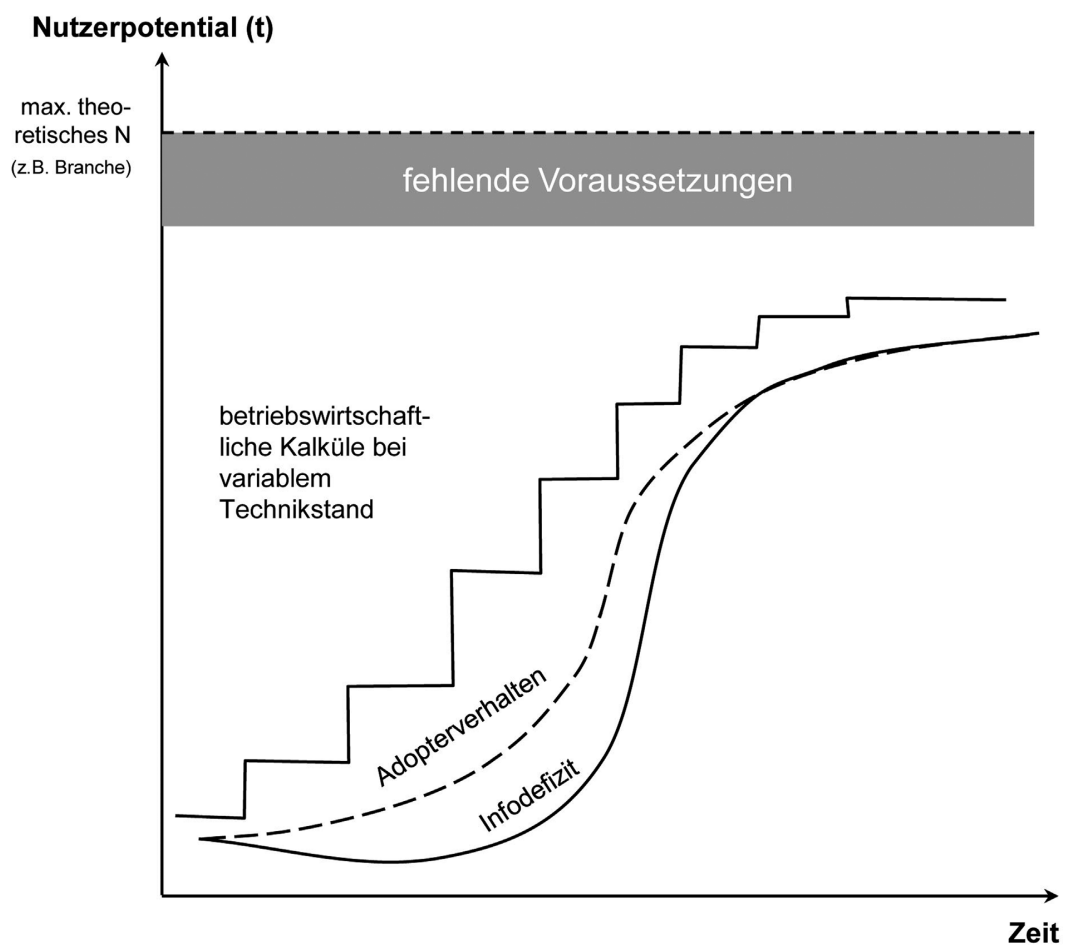

Abb. 2 | Ermittlung realistischer Adopterpotentiale (aus Dreher I997, 237).

neuen Gütern, neuen Methoden, neuen Organisationsformen und neuen Märkten die ,kapitalistische Maschine، in Bewegung setzen und halten. Schumpeter unterstellte dem I9II noch als Person verstandenen Unternehmer nicht nur Gewinnstreben, sondern auch den Willen zur Macht, Prestigedenken und Schaffensfreude..$^{8}$ Neben diesem, dem neoklassischen Verständnis gegenüber erweiterten Motivset besteht das rationale Kalkül in einer möglichen, gegebenenfalls nur zeitlich befristeten Monopolstellung des innovativen Unternehmers, bis Imitatoren die Neuerungen im Wirtschaftsprozess übernommen und damit einen nivellierten, bis zur nächsten Innovation statischen Zustand hergestellt haben.

Im Zuge seiner weiteren theoretischen Arbeiten überträgt Schumpeter I942 die Rolle des innovativen Unternehmers auf Unternehmen insofern, als er größeren Unternehmen eine stärkere Innovativkraft zumisst als kleinen. Hintergrund dieser Überlegung ist, dass wichtige Innovationen nur noch mit einem erheblichen Aufwand an Forschung und Entwicklung realisiert werden können. Die großen Unternehmen, die zu solchen Ausgaben

58 Bollmann $1990,45 \mathrm{ff}$. 
fähig sind, übernehmen damit die Rolle der innovativen Unternehmer. Mit diesen Überlegungen und Hypothesen legte Schumpeter die Grundlage für viele Forschungsarbeiten, die auf der Suche nach der Bestätigung oder Falsifizierung seiner Thesen waren. Mit der Bezeichnung des Charakters des kapitalistischen Prozesses als „evolutionär“59 gab er der Forschungsrichtung, die sich mit der Dynamik des Innovationsgeschehens befasst, ihren Namen.

\section{Grundsätze der Evolutionstheorie}

Die evolutionstheoretische Innovationsforschung sieht sich bewusst im Gegensatz zum neoklassischen Wirtschaftsverständnis. Einigkeit in den verschiedenen Richtungen der evolutorischen Innovationsforschung besteht in der Ablehnung der Gewinnmaximierungs- und Gleichgewichtshypothese und in der Betonung des dynamischen Entwicklungsgedankens. Da das dynamische Wesen der Wirtschaft dominiert, wird dem neoklassischen Verständnis ein aus der Biologie entlehntes, evolutorisches entgegengestellt. Begriffe wie Selektion, Reproduktion und Mutation werden für Beschreibungen ökonomischer Strukturen und Systeme verwendet. ${ }^{60}$ An diesem Punkt hören die Gemeinsamkeiten der Vertreter dieser Forschungsrichtung auf. Die bloße Analogiebildung zur Biologie wird von der Mehrheit der Autoren als unnötige Einschränkung angesehen. ${ }^{6 r}$ So werden beispielsweise Mutation und Adoption nicht unbedingt als zufällige Ereignisse, sondern als Ergebnisse von Suchprozessen von Individuen charakterisiert. Schwitalla teilt die evolutionstheoretische Schule in eine individualistisch-behavioristische und in eine institutionell-historische Richtung auf. ${ }^{62}$ Während die erstere die Erklärung wirtschaftlicher und gesellschaftlicher Phänomene auf der Basis von individuellem Verhalten bzw. vom Verhalten von Organisationen vornimmt, erklärt die institutionell-historische Richtung das Verhalten von Systemen und das Zusammenspiel mit der Umgebung. Bestandteil der individualistisch-behavioristischen Innovationserklärungen sind Produktlebenszyklusmodelle sowie die Simulationsmodelle von Nelson und Winter. ${ }^{63}$ Vertreter einer institutionellhistorischen Innovationsforschung verknüpfen empirische Beobachtungen und Schumpetersche Erklärungsmuster mit dem Kondratieffschen Modell der langen Wellen der wirtschaftlichen Entwicklung.

59 Schumpeter $1942,136$.

60 Vgl. Schwitalla I993, 39-40.

6I Z.B. Witt I987, 88; von Hayek I969, I42.

62 Schwitalla I993.

63 Nelson/Winter 1982. 
In Erweiterung von Schumpeters Überlegungen differenziert Heuss den bei Schumpeter nur als Antriebskraft gedachten innovativen Unternehmer ${ }^{6}$ weiter und entwickelt eine Typologie, die die Phasen eines Marktzyklus anhand der jeweils unterschiedlichen Zusammensetzung der Unternehmerschaft erklärt. Er unterscheidet zwischen Pionierunternehmern, spontan imitierenden, unter Druck reagierenden und immobilen Unternehmern. Diese vier Typen dominieren jeweils in einer Phase des Marktzyklusses. In der Experimentierphase sind es die Pionierunternehmer, die neue Produkte oder Prozesse entwickeln. Dieser Phase folgt eine Expansionsphase. Hierbei treten die spontan imitierenden Unternehmen in Erkenntnis möglicher Chancen an die Seite der Pionierunternehmen und fördern eine weitere Marktausweitung. Die sich anschließende Ausreifungsphase ist durch ein Abnehmen des Nachfragewachstums gekennzeichnet, bei dem die Unternehmen nur noch geringe Möglichkeiten haben, die Preise und Kosten zu variieren. In dieser Phase geraten die unter Druck reagierenden Unternehmer in den Zwang, eigene Prozessverbesserungen durchzuführen und Produktverbesserungen zu übernehmen. Die immobilen Unternehmer beherrschen in einer Stagnationsphase den Markt und versuchen durch Marktmacht, das Aufkommen neuer innovativer Unternehmer zu unterbinden.

In Anlehnung an diese Modellvorstellung versuchen Abernathy und Utterback die Produktlebenszyklus-Ansätze auszubauen, indem sie zwischen Produkt- und Prozessinnovationen unterscheiden und deren Entwicklungsgeschichte mit den Maßnahmen der Unternehmen verbinden. ${ }^{65}$ Demnach wird zu Beginn der Entwicklung einer Produktlinie nach einer neuen technischen Ausführung mit einer besseren Funktionserfüllung gesucht. Der Innovationsgrad wäre bei Realisierung der Innovation vergleichsweise hoch und in der Anfangsphase führen die Anforderungen der Pilotanwender zu häufiger Variation der Neuerung. Kennzeichen dieser Phase ist ein Wettbewerb über die Funktionsfähigkeit und Qualität des neuen Produkts. Hat sich ein so genanntes dominantes Design im Zuge der Marktentwicklung ergeben, bildet dieses einen Standard für weitere Produktentwicklungen. Nach diesem Durchbruch wird der Wettbewerb zunehmend über den Preis ausgetragen. In der Übergangsphase nehmen die Neuerungen am Produkt ab bzw. sind marginale Produktverbesserungen und keine Erweiterungen des Funktionsumfangs. Wächst der Markt weiter und damit die Sicherheit der Anwender über die Erwartungen an das Produkt, müssen die Anbieter zunehmend auf die Effizienz ihres Herstellungsprozesses achten, da der Wettbewerb über den Preis ausgetragen wird. Prozessinnovationen gewinnen dabei an Bedeutung. Dieser Zyklus ist nur zu unterbrechen, wenn durch frühzeitige Entwicklung einer neuen Sprunginnovation eine Ausbruchsstrategie ermöglicht wird. ${ }^{66}$

64 Heuss i965, I5.

65 Abernathy/Utterback 1978; 1982.

66 Vgl. auch Christensen I997. 
Auf der Basis der oben skizzierten Ansätze, in denen den Unternehmen unterschiedliche Verhaltensweisen (behaviours) unterstellt werden, sowie der Erkenntnisse aus organisationstheoretischen Ansätzen ${ }^{67}$ gehen Nelson und Winter in ihrem Modell von folgender Charakterisierung der Unternehmen aus: Unternehmen befinden sich ständig auf der Suche nach Möglichkeiten, ihre Gewinne zu verbessern. Die Möglichkeiten ergeben sich aber aus den Erfahrungen und Verhaltensweisen zu vorherigen Zeitpunkten, die die Unternehmen in den Besitz bestimmter Fähigkeiten gebracht haben. Im Zuge dieser Lern- und Entwicklungsprozesse sowie zufälliger Ereignisse besteht die Tendenz, dass erfolgreichere Unternehmen weniger erfolgreiche vom Markt verdrängen. ${ }^{68}$ Nelson und Winter differenzieren das Unternehmerverhalten in Routine und Innovation: Mit Routine sind diejenigen Verhaltensweisen einer Unternehmung gemeint, die die Unternehmeraktivitäten in geregelten Bahnen verlaufen lassen. Sie dienen als Voraussetzung für ein wenig störanfälliges Funktionieren. Nelson und Winter sehen dies als Analogie zur Gen-Struktur eines Lebewesens. In dieser Analogie fortlaufend sind Mutationen diejenigen Innovationen, die entstehen, wenn durch Selektion am Markt die Notwendigkeit, sich einer veränderten Umwelt anzupassen, zunimmt und aufgrund von Suchprozessen neue Lösungen entstehen. Im Unterschied zur Biologie können entsprechende Suchstrategien, z. B. durch ein bestimmtes Budget für Forschung und Entwicklung, die Wahrscheinlichkeit für eine erfolgreiche Suche beeinflussen. Nelson und Winter erstellen auf der Basis dieser Überlegung ein formales Modell, das den dynamischen Prozess aufzeigt und Verhaltensmuster von Unternehmen und Marktergebnisse im Zeitverlauf bestimmt.

Zentral bei diesem Modell ist die Erkenntnis, dass in der Analogie zum biologischen Entwicklungsprozess Anstöße zur Entwicklung des Systems aus einer eigenen Dynamik heraus entstehen, aber die Suchanstrengungen der Unternehmen und Anpassungsvorgänge sich in einem langsamen, kontinuierlichen Ablauf vollziehen und nicht das Ergebnis von technischen Revolutionen oder vom Himmel gefallener Innovationen sind. Zwar erklären auch Nelson und Winter den technischen Fortschritt noch exogen. Dies geschieht aber nicht auf deterministische Weise, sondern durch eine Wahrscheinlichkeitsverteilung, die durch die individuellen Suchanstrengungen der Unternehmen sowie durch die auf den Märkten anzutreffenden ökonomischen Bedingungen beeinflusst wird.

67 Cyert/March i963 und Simon I959.

68 Nelson/Winter I982, 4I-2I. 
Die von Schwitalla vorgenommene Klassifikation ordnet diejenigen Innovationsforscher in die in der Überschrift genannte Kategorie ein, die auf der Basis der historischen Betrachtung der technischen und sozioökonomischen Strukturentwicklung qualitative Erkenntnisse auf der Basis vorhandenen Datenmaterials entwickeln. ${ }^{6}$ Da diese Typologien und Klassifikationen meistens vom tatsächlichen Technologieverlauf abgeleitet werden, ist hier die Abgrenzung zur empirischen Forschung schwierig.

Dieser Betrachtungsansatz wirft den anderen Ansätzen zur Erklärung des technischen Wandels vor, das Langzeitverhalten und deren entscheidende Elemente zu vernachlässigen. Besonderer Wert wird bei diesem Ansatz dem Zusammenspiel des sich ökonomisch verhaltenden Unternehmens mit der Umgebung, den Ausgangsbedingungen und den jeweiligen Lernprozessen im Institutionengefüge beigemessen. ${ }^{70}$

Ausgangspunkt der Betrachtung waren die Erkenntnisse von Freeman, dass die Forschungs- und Entwicklungstätigkeit in den westlichen Volkswirtschaften zunehmend von ausgebildeten Wissenschaftlern und Ingenieuren in den industriellen FuE-Einrichtungen betrieben wird.7 Die Ergebnisse dieser FuE-Projekte bilden einen so genannten Erfindungskomplex, da die frühere Unterscheidung von Produkt- und Prozessinnovation in vielen industriellen FuE-Prozessen kaum noch als trennende Charakteristik verwendet werden kann. Die Gründe für dieses professionalisierte FuE-System sind der zunehmend wissenschaftliche Charakter der Technologien, ihre Komplexität sowie der allgemeine Trend zur Arbeitsteilung und Spezialisierung.72

Die mikroökonomische Fundierung des institutionell-historisch erklärten technischen Wandels

Dosi versucht in seinem grundlegenden Artikel die Fundierung der obigen Beobachtungen auf mikroökonomischer Ebene zu erreichen. ${ }^{33}$ Zentrales Problem ist für ihn dabei, wie Wissen und Fähigkeiten von den Unternehmen gesammelt werden, um technologische Probleme zu lösen. Diese Lernprozesse vollziehen sich auf der Basis des technologischen Wissens und sozioökonomischer Rahmenbedingungen. Diese Determinanten definieren so genannte technologische Paradigmen. Auf Basis dieser Paradigmen bewegt sich der technische Fortschritt auf so genannten technologischen Bahnen (trajectories). Die das technologische Paradigma beeinflussenden Determinanten sind in der Abbildung 3 dargestellt.

69 Vgl. Schwitalla I993, 60.

70 Vgl. im Überblick Dosi et al. I988.

7I Freeman I982.

72 Vgl. Schwitalla 1993, 62.

73 Dosi 1988. 


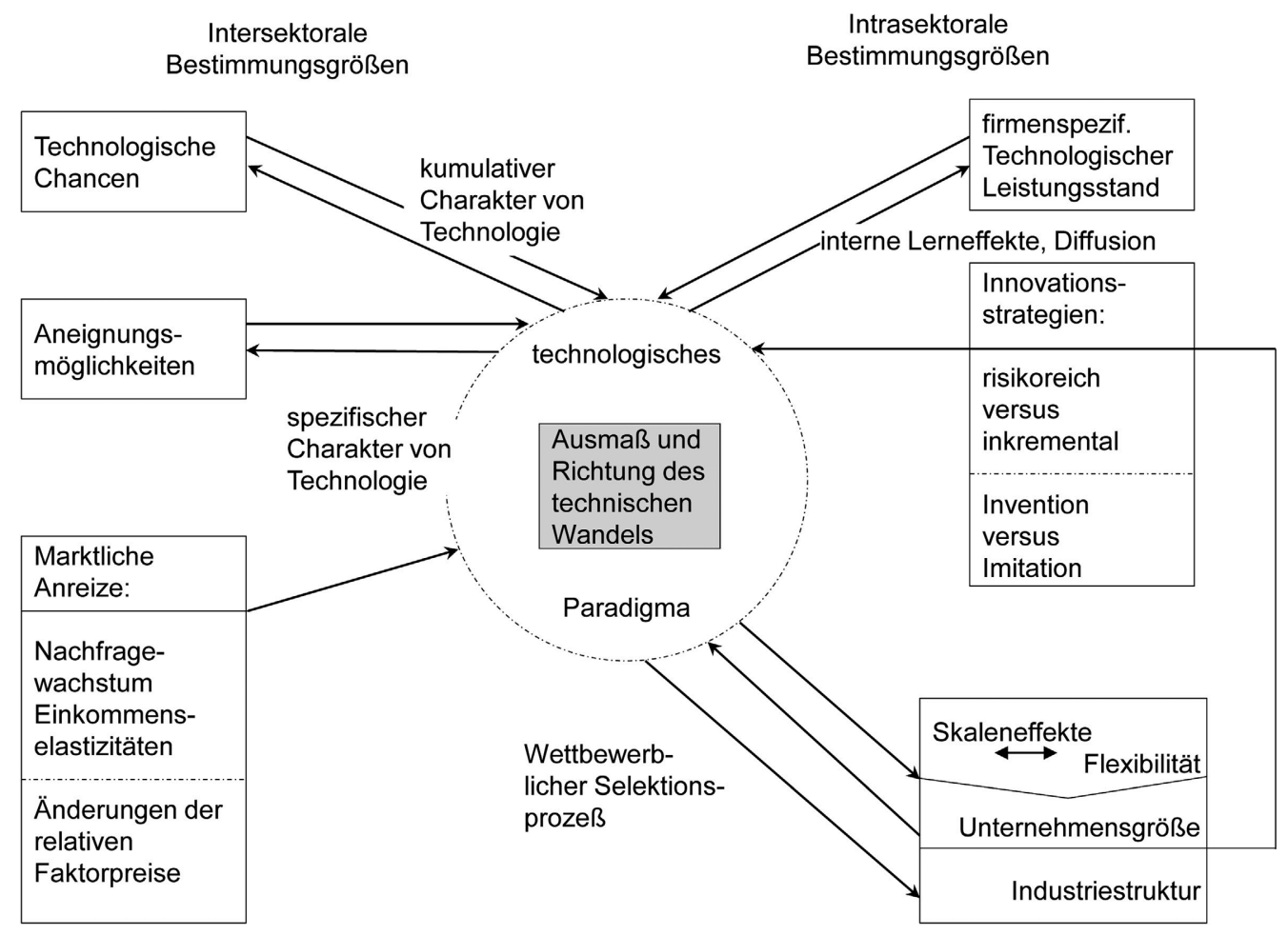

Abb. 3 | Determinanten inter- und intrasektoraler Innovationsmuster nach Dosi I988 (aus Schwitalla I993, 65).

Dosi hat versucht, diese Überlegungen zu formalisieren, was ihm zusammen mit L. Orsenigo und G. Silverberg gelungen ist.74 Das Modell zeigt, dass in der dynamischen Interaktion zwischen der makro- und mikroökonomischen Ebene das System notwendigerweise zu räumlich und zeitlich unterschiedlichen Verhaltensweisen kommen muss. Diese lassen sich aus den Unterschieden auf der mikroökonomischen Ebene herleiten. Die Beobachtung ist dann wichtig, wenn man langfristige empirische Gleichmäßigkeiten bei der Verbreitung von Techniken betrachten möchte. Von der Makroperspektive aus sind Regelmäßigkeiten der evolutionären Entwicklung wie z.B. S-förmige Diffusionsverläufe eher die Konsequenz aus der Vielfalt der Erwartungen, Wahrscheinlichkeiten und Verhaltensweisen der ökonomischen Akteure75 als die Folge gleicher Unternehmensverhaltensweisen oder -maximen.

75 Vgl. Grübler I992, 38. 


\section{Elemente einer dynamischen Innovationsökonomie}

\section{Neue Entwicklungen}

In den letzten beiden Jahrzehnten haben sich vor allem aus der evolutionären ökonomischen Innovationsforschung wichtige Beobachtungen zur neuen Qualität von Innovationsphänomenen sowie von unternehmerischen und gesamtwirtschaftlichen Innovationsprozessen ergeben. ${ }^{6}$ In den letzten Jahren hat sich diese Innovationsforschung auf das Konstrukt des ,Innovationssystems ‘ und die Erforschung des institutionellen Wandels konzentriert. Die empirische Erforschung betrieblichen Innovationsverhaltens hat sich dagegen nach ersten Überblickstudien77 eher auf Einzelelemente beschränkt, die etwa nach Phasen von Innovationsprozessen, nach Innovationszielen oder nach wichtigen Ressourcen und Kompetenzen strukturiert sind. So lassen sich drei Hauptbefunde isolieren, die entsprechende Niederschläge in den erwähnten Forschungsgebieten finden:

- Veränderungen im Charakter der Innovationen,

- Innovationstätigkeiten entlang von Entwicklungsbahnen,

- Bedeutung des gesellschaftlichen Kontextes für Innovationen.

Der Charakter der Innovation hat sich in den Betrachtungen erheblich geändert. Stand zunächst der Erfinder und Schumpetersche Unternehmer als Innovator im Mittelpunkt, so wurde dann von einem linearen Ansatz, einer Kette von Forschung, Entwicklung bis hin zum Markteintritt der Neuerung gesprochen. ${ }^{7}$ Ergänzt man die Beobachtungen zur Bedeutung der Forschung und Wissenschaft79 zur Bedeutung von Zulieferstrukturen und Interdependenzen innerhalb und zwischen Branchen ${ }^{8 \circ}$ sowie zur Relevanz der Kunden und ihres Einflusses auf Innovationserfolg und -prozess, ${ }^{8 \mathrm{I}}$ so zeigt sich, dass die Vorstellung von der ,einfachen' Kette von Innovationsschritten nicht ausreicht. Deshalb hat sich die Innovationsforschung von dem linearen Ansatz gelöst. Zum Beispiel spricht Rothwell anhand von Beobachtungen der Konsequenzen der Informationstechnik für Innovationsprozesse vom „Fifth Mode of Innovation“82 oder Kline und Rosenberg sprechen vom „chain-link-Modell“83, in dem die Verknüpfung von Schritten des Innovationsprozesses durch Feedback-Schleifen vorgenommen wird. Zusammengefasst betont man heute:

- die Bedeutung der Wissensbasis für Innovationen,

- die Lernfähigkeit von Institutionen, insbesondere Unternehmen,

76 Übersichten in Freeman/Soete 1997; Grupp 1997.

77 SAPPHO-Projekt oder, in Deutschland: Maas I990.

78 Etwa klassische Definition der OECD I997, nach der sich die amtlichen Statistiken ausrichten.

79 Freeman/Soete I997.

80 Pavitt I984.

8I von Hippel I988.

82 Rothwell I992.

83 Kline/Rosenberg 1986. 
- die Interaktionen in Form von Feedback-Schleifen,

- die Innovationsgeschwindigkeit als kritisches Element der Innovationsfähigkeit und

- die strategische Zusammenarbeit von Partnern und Kunden in Netzwerken.

Damit wird nachvollziehbar, wie komplex und kompliziert betriebliche Innovationsprozesse geworden sind. Fragen des Ressourcenmanagements, insbesondere der ,weichen' Faktoren, wie Kompetenzen, Wissen etc. sowie der Zusammenarbeit über betriebliche Grenzen hinaus, ${ }^{84}$ um Kompetenzen zusammenzuführen und Risiken zu streuen, stehen verstärkt im Vordergrund. Hier wird von den meisten Quellen auf fassbare Dimensionen von Wissen wie Patente oder Lizenzen rekurriert - wohlwissend, dass Erfahrungswissen, Einbeziehen von Betroffenen etc. entscheidende Bestandteile betrieblicher Kompetenzen sind. ${ }^{85}$

Die Zusammenarbeit mit Partnern, Nutzern, Zulieferern etc. kommt als Quelle von Innovationsideen und zur besseren Nutzung von Kompetenzen erhebliche Bedeutung zu. Daher sind die Zusammenarbeit im Rahmen von FuE-Kooperationen, Fragen der Wissensaneignung (absorptive capacity) und der Aufnahme von Kundenwünschen bis hin zu einer offenen Form der Generierung von Innovationen wichtige Themen des Innovationsmanagements.

Als weiterer Debattenstrang wird der Ansatz der ,Routinen`86 zu Hilfe genommen, um Schritte im Innovationsprozess zu verbessern. Veränderungen werden als Lernprozesse verstanden, die zukünftig die Anpassungsfähigkeit steigern sollen. Dabei wird ein umfassender Prozess von der Idee über die Umsetzung von Marktsignalen bis zur Marktdurchdringung zugrunde gelegt, der durch Nichtlinearität, Rückkopplungsschleifen, Dominanz von Management- und Organisationsfaktoren und externer Zusammenarbeit gekennzeichnet ist. Dies wird recht griffig durch den Ausdruck der „innovation journey“87 beschrieben.

Diese Ansätze gelten zuvorderst als Elemente einer pfadabhängigen inkrementellen Innovationsstrategie. Für disruptive Innovationen werden nicht nur Fragen der Früherkennung neuer Techniken zur Verbesserung der Reaktionsfähigkeit diskutiert, ${ }^{88}$ sondern vor allem der Umgang von Unternehmen mit solchen schockähnlichen Entwicklungen. ${ }^{89}$ Kern ist dabei die Beobachtung von Christensen, dass die vorhandenen Organisationsstrukturen einer Unternehmung auf den Innovationspfad eingestellt sind. $9^{\circ}$ So müssen sich bestehende Organisationsstrukturen entweder extrem anpassen ${ }^{91}$ oder die Herausfor-

84 Grundlagen gesammelt bei Teece 2003, konkret z. B. die Aufsätze zum Technologiemanagement in Burgelman et. al. 2004, deutschsprachig am besten derzeit durch Gerybadze 2004 und Hauschildt 2004.

85 Vergleiche hierzu Tidd et al. 200I zu "tacit knowledge“ und "creating an innovative organisation".

86 Tidd et. al. 200I.

87 van de Ven et. al. I999.

88 Herstatt/Verworn 2003.

89 Arnold 2003.

90 Christensen 1997.

9I Francis/Bessant 2005, als paradigmatischen Wandel durch Wettbewerb über „neue Geschäftsmodelle“. 
derungen werden außerhalb der bestehenden Strukturen als corporate venturing-Aktivitäten, Aus- oder Neugründungen, in Angriff genommen..$^{2}$

Die zweite Beobachtung betrifft die Entwicklung von technischen Innovationen über die Zeit: Innovationstätigkeiten finden auf bestimmten, i.d.R. auf technischen Basisentwicklungen beruhenden Pfaden (trajectories) statt.93 Ein Wechsel von diesen Pfaden in neue Bahnen wird auf Unternehmensebene als disruptive Innovation, eine Weiterentwicklung entlang des Pfades als inkrementelle oder bei Sprüngen in der vorhandenen Technologie als radikale Innovation bezeichnet.94 Diese Entwicklungsbahnen erwachsen aus neuen Paradigmen, die sich aus einer Melange aus technischen Entwicklungen und sozialen wie wirtschaftlichen Rahmenbedingungen bilden. Dabei wird die Technikentwicklung, die anfangs durch Vielfalt gekennzeichnet ist, im Laufe der Zeit durch Selektion zu einem dominanten Design verdichtet. Dabei spielen nicht nur Marktmechanismen, sondern auch soziale Präferenzen eine erhebliche, wenn nicht gar die wichtigste Rolle95, die dazu führen können, dass sich auch technisch weniger leistungsfähige Lösungen durchsetzen. Die Entwicklungsbahnen sind zu späteren Zeitpunkten von den Unternehmen kaum oder gar nicht mehr zu verlassen, da die dafür notwendigen Transaktionskosten nicht (mehr) aufgebracht werden können. Man spricht dabei vom Lock-In-Effekt oder Pfadabhängigkeit von Entwicklungen.

Zusammen gesehen unterstreichen sowohl die Intensivierung der überbetrieblichen Zusammenarbeit als auch die sozio-technischen Paradigmen, die die Innovationsbahnen prägen, die Abhängigkeit der Unternehmen von ihren gesellschaftlichen Kontexten, wie Abnehmern, FuE-Partnern, Zuliefererstrukturen, Ausbildungssystem, anderen regulatorischen Rahmenbedingungen etc. Dabei wird dieser Kontext für betriebliche Innovationen nicht nur als Quelle für Ressourcen, sondern auch als Nachfrager und Richtungsweiser für Innovationen angesehen. In der Gesamtschau wird daher von Innovationssystemen gesprochen, in denen die Unternehmen mit ihren Innovationstätigkeiten eingebettet sind und die national, regional oder sektoral analysiert werden können. ${ }^{6}$

\section{Innovationssysteme}

Nach Grupp et al.97 umfassen „Innovationssysteme - nationale, regionale, sektorale - nach international akzeptiertem Verständnis die ,Kulturlandschaft‘ all jener Institutionen, die wissenschaftlich forschen, Wissen akkumulieren und vermitteln, die Arbeitskräfte ausbil-

92 Burgelman 1984.

93 Dosi 1982

94 Christensen I997.

95 Vgl. auch entsprechende Ansätze der Soziotechnik: Callon I995, oder zur Innovationskultur: Wengenroth 200I.

96 Lundvall I992, Edquist I997, Malerba 2004, auch Porter I990.

97 Grupp et al. 2004, 87 f. 
den, die Technologie entwickeln, die innovative Produkte und Verfahren hervorbringen sowie verbreiten; hierzu gehören auch einschlägige regulative Regimes (Standards, Normen, Recht) sowie die staatlichen Investitionen in entsprechende Infrastrukturen..$^{8}$ Innovationssysteme erstrecken sich also über Schulen, Universitäten, Forschungsinstitute, forschende Unternehmen in der Industrie, politisch-administrative Instanzen sowie Netzwerke zwischen diesen Akteuren. Sie sind ,hybrid' und repräsentieren damit einen Ausschnitt der Gesellschaft, der weit in andere Bereiche hinein strahlt: Innovationssysteme prägen die Modernisierungsprozesse einer Gesellschaft entscheidend.“

Unter dem Begriff „Nationales Innovationssystem“ (NIS) wurde lange nur die Forschungs- und Entwicklungsinfrastruktur eines Landes verstanden. In den 9oer Jahren wurde zunehmend zur Kenntnis genommen, dass neben den Universitäten und Forschungseinrichtungen auch staatliche, halbstaatliche und private Institutionen zur Finanzierung, Durchführung und Regulierung Bestandteile sind. Dabei sind aber nicht nur die Institutionen, sondern auch ihre Interaktionen im Innovationssystem von Interesse. 99

Es lassen sich die Herausforderungen folgendermaßen charakterisieren:

- eine Erweiterung der Innovationssystem-Sicht ausgehend von der Forschungs- undEntwicklungstätigkeit

- einerseits auf die wachsende Bedeutung der Wissensproduktion ${ }^{\text {Ioo }}$ sowie

- andererseits auf erfolgreiche Marktumsetzung bzw. Nachfrageinduzierte Innovationen durch günstige Marktbedingungen (bis hin zu ,Vorreitermärkten') ${ }^{\mathrm{IOI}}$,

- die Nutzung von disruptiven Erfindungen, die neue Pfade eröffnen, als Grundlage für Wachstumschancen in der Volkswirtschaft.

Der Umbruch des Innovationsgeschehens in den Unternehmen führt zu einem neuen Modus der Wissensproduktion. ${ }^{102}$ Er verwirft den eher linearen, an Fachdisziplinen gebundenen, auf Binnenstrukturen bezogenen Ansatz der Wissensproduktion, wie Grundlagenforschung, angewandte Forschung und Entwicklung, dem die deutsche Entwicklung zugrunde lag. Vielmehr müssen konventionelle Trennungen überwunden werden. Der neue Modus ist charakterisiert durch Inter- und Transdisziplinarität von Forschung und Entwicklung. Langfristige anwendungsorientierte Grundlagenforschung wird für viele Technikgebiete zunehmend stärker gewichtet (z. B. für die Lebenswissenschaften) und Vielfalt und Wettbewerb werden als wesentliche Bedingung für die Entwicklungsfähigkeit eines Innovationssystems gesehen. ${ }^{103}$

Haben sich somit neue grenzüberschreitende und lernorientierte Interaktionsmechanismen und wettbewerbsorientierte Akteursvielfalt von Wirtschaft, Staat und Wissenschaft

98 Freeman I987; Lundvall I992; Edquist I997: nach Grupp et al. 2004.

99 Meyer-Krahmer 200I, Lundvall et. al. 2002.

I00 Coombs/Hull I998; Schmoch et al. 2000.

IOI Beise 2OOI.

IO2 Gibbons et al. I994.

I03 Zur Messung s. Grupp I997. 
als wichtige Elemente der Wissensproduktion in einem Innovationssystem festmachen lassen, ${ }^{\text {I04 }}$ so zeigt sich ebenfalls die Bedeutung der marktseitigen Erweiterung des Innovationssystems. ${ }^{\text {I05 }}$

Da Lernprozesse und Feedbackschleifen zentrale Elemente geworden sind, ist die Einbindung entsprechender Akteure im Innovationssystem, die sich mit der Markteinführung, Produktion, Nutzung und Entsorgung befassen, für die Realisierung von ,pull-Mechanismen` von Nachfrageseite zentral. Diese Mechanismen können - bei entsprechend intelligenten Ansätzen - mittels Schaffung neuer Rahmenbedingungen und Ausnutzung vorhandener Stärken zur Schaffung von Vorreitermärkten genutzt werden. ${ }^{\circ 06}$ Dabei gilt es vor allem die Koordination der verschiedenen Politikbereiche sicherzustellen. Auch das Durchhaltevermögen der politischen Akteure ist zu stärken, das angesichts neuer Gewinner und Verlierer im Innovationswettbewerb zu erbringen ist. ${ }^{107}$

Neben diesen, die Grundlagen und Ausdehnung des Innovationssystems betreffenden Aspekten gilt es die Bereiche zu identifizieren, in denen das Innovationssystem für das Wirtschaftswachstum die wichtigste Rolle spielen kann. Dies sind vor allem diejenigen, in denen disruptive Innovationen (in diesem Kontext auch Basisinnovationen oder Makro-Erfindungen genannt) stattfinden.

\section{Trajektorien}

Zur Skizzierung einer Modellvorstellung solcher Entwicklungsverläufe von Basisinnovationen kann auf drei wichtige Diskussionsstränge zurückgegriffen werden: Zum Ersten sind es die erfahrungsbasierten Darstellungen der Techniker und Ingenieure zu Technikentwicklungen und Lernkurven, ${ }^{108}$ zum Zweiten die Ansätze aus den vorrangig angel-

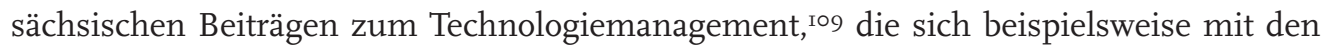
Schwerpunkten von Innovationsanstrengungen im Zeitverlauf beschäftigen. ${ }^{\text {II }}$ Zum Dritten können wichtige Befunde aus der historisierenden und evolutionären Innovationsforschung herangezogen werden. So können Innovationszyklen beispielsweise mit Akteursgruppen, ${ }^{\text {III }}$ Wettbewerbsbedingungen, ${ }^{\text {II2 }}$ Beziehungen zum nationalen und sektoralen

I04 Etwa in ihrem Modell der verschränkten Dreier-Helix von Wirtschaft, Staat und Wissenschaft: Etzkowitz/ Leydesdorff 2000.

I05 Beise 200I.

I06 Beise 200I.

I07 Meyer-Krahmer 2004.

Io8 Weule 2002; beispielhaft für Oberflächentechnik: Rickerby/Matthews I99I.

I09 Vgl. Übersichten in Tidd et al. 200I; Reger/Schmoch, I996

IIo Utterback/Abernathy I975; Stumpfe o.J.; Mauri/McMillan I999.

III Klepper I996.

II2 Werker 2003. 
Innovationssystem, ${ }^{\mathrm{II} 3}$ Interdependenzen zur Technikgenese und Wissenschaft ${ }^{\mathrm{II} 4}$ sowie mit der Herausbildung von Entwicklungsbahnen und dominanten Designs ${ }^{\text {II5 }}$ in Verbindung gebracht werden. Letztlich gehören die schon älteren Modelle zur Diffusion von Innovationen ebenfalls in diese Kategorie. ${ }^{\mathrm{II} 6}$

Meyer-Krahmer und Dreher fassen die Literatur beispielhaft in einem 6-Phasen-Modell zusammen ${ }^{\mathrm{II} 7}$ (vgl. Abb. $4^{\mathrm{II} 8}$ ). Die Dimensionen stellen das Aktivitätsniveau (gemessen mittels unterschiedlicher Indikatoren), die Breite der Forschungsaktivitäten beziehungsweise der angestrebten Nutzungsmöglichkeiten sowie die Zeit dar.

Nach der Entdeckung und Exploration (Phase I) werden die neuen technischen Prinzipien und wissenschaftlichen Lösungen auf breiter Front erforscht und transdisziplinär auf andere Fragestellungen übertragen und bearbeitet. Dies erzeugt in einer breiter werdenden Gemeinschaft der Wissenschaftler und Forscher ein euphorisches Gefühl über die Möglichkeiten der neuen Entwicklung (Phase 2). Im Laufe der Zeit erweisen sich aber einzelne Optionen als entweder wissenschaftlich oder wirtschaftlich nicht realisierbar, so dass Forschungsaktivitäten eingeschränkt oder gar eingestellt werden müssen (Phase 3). Letztlich führt dies zur Verunsicherung, bei der nur entweder diejenigen Akteure mit dem längsten Atem oder die mit radikal neuen Ansätzen zu einer Neuorientierung der Entwicklung beitragen (Phase 4). Sie erzielen die entscheidenden ersten industriellen Durchbrüche, von denen diejenigen, die sich am Markt am schnellsten durchsetzen, den zukünftigen Umgang mit der Basisinnovation prägen (dominante Designs) (Phase 5). In der Diffusionsphase (Phase 6) fächern sich die Anwendungsmöglichkeiten wieder auf, da Skaleneffekte zur Verbilligung der Lösungen führen und damit neue Einsatzgebiete erschlossen werden können.

Jeder Phase können typische Akteure, betroffene Unternehmensbereiche und Indikatoren zugeordnet werden. ${ }^{\text {II }}$

Aufgrund der damit verbundenen Friktionen ergeben sich im Zuge solcher Zyklen die stärksten Wachstumschancen - vorausgesetzt aufnahmebereite Vorreitermärkte stehen bereit und das Innovationssystem passt sich in allen Bereichen schnell an. ,Gewinnen“ wird das anpassungsfähigste Innovationssystem. Allerdings sind diese Phasen von erheblichen Umbrüchen gekennzeichnet, die Verlierer und Gewinner aufweisen und Anpassungsprozesse erfordern. ${ }^{\text {I20 }}$

II3 Edquist I997; Archibugi/Lundvall 200I, national: Meyer-Krahmer 200I; sektoral: Malerba 2002; regional: Dalum et. al. 2002.

II4 Coombs/Hull I998;Tijssen 2002 Schmoch et. al. 2000.

II5 Dosi I998; Cainarca et. al. I992 vergleiche auch Kapitel 2.

II6 Rogers I983, Geroski 2000.

II7 Meyer-Krahmer/Dreher 2004.

II8 Phasenbezeichnungen ähnlich zu Grupp I997.

II9 Vgl. im Einzelnen Meyer-Krahmer/Dreher 2004.

I20 Meyer-Krahmer 2004. 


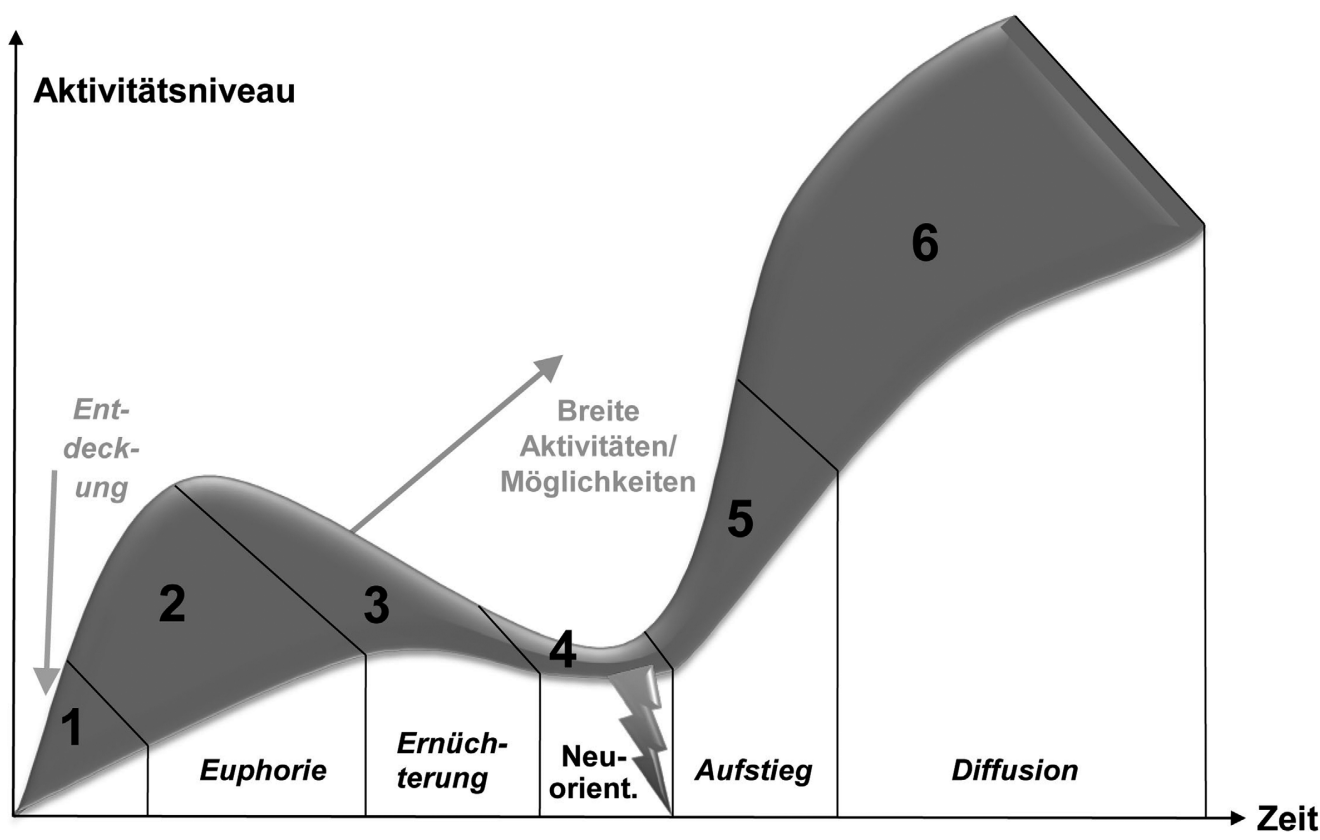

Abb. 4 | Innovationszyklus-Modell (schematische Darstellung).

Die Attraktivität eines Innovationssystems ist damit weniger von statisch-komparativen Wettbewerbsfaktoren wie Löhnen oder Kosten bestimmt, sondern von seiner ,dynamischen Effizienz', d.h. von seiner Fähigkeit sich zielgerichtet auszurichten. Diese wiederum ist weitgehend vom Ausmaß der strategischen Intelligenz ${ }^{\text {I2I }}$ beim Finden und Durchsetzen neuer Strukturen und Märkte abhängig. Die Ähnlichkeit zum „Wettbewerb der Geschäftsmodelle“ bei disruptiven Innovationen auf betrieblicher Ebene ist nicht zufällig. Auch hier ist das schnelle Rearrangement der Ressourcen und Kompetenzen zentral. Hier wie dort stellen gerade diese Umbruchphasen die Möglichkeit dar, sich auf einem neuen Pfad erfolgreich und dauerhaft zu positionieren.

I2I Strategic Intelligence ist ein Sammelbegriff für die unterschiedlichen Instrumente und Methoden, die angewendet werden, um systematisch Informationen für die Konzeption und Planung in der Wissenschafts-, Forschungs- und Innovationspolitik bereit zu stellen. Im Kern handelt es sich dabei um Methoden im Bereich Vorausschau, Evaluation und Technikbewertung; Grupp et. al. 2004, 92. 


\section{Zusammenfassung}

In diesem Beitrag sollten die wichtigsten Erkenntnisse und Modelle der Wirtschaftswissenschaften zur Verbreitung von Innovationen und der Determinanten der Verbreitungsprozesse erfolgen, um die interdisziplinäre Perspektive auf Fragen zur Diffusion von Neuerungen im Rahmen des Exzellenzclusters Topoi zu komplettieren.

Ausgehend von den ,Klassikern' der ökonomischen Diffusionsforschung werden die Modellfamilien kurz skizziert. Die grundlegenden Arbeiten von Mansfield gelten als informationsbasierte Ansätze, bei denen die Geschwindigkeit der Informationsverbreitung sowie die Höhe des Stimulus durch die unterschiedliche Wirtschaftlichkeit die Verbreitung über die Zeit bestimmen. Die ProBit-Ansätze gehen im Gegensatz dazu von Adoptern mit heterogenen Eigenschaften aus, die bei vollständiger Information aufgrund ihrer individuellen Charakteristika und der Kostendegressionseffekte eine rationale Adoptionsentscheidung treffen. Gemeinsam ist beiden Ansätzen, dass die empirisch nachweisbaren S-Kurven nachgebildet werden können.

Die Weiterentwicklungen betrafen die mathematischen Eigenschaften der Modelle, die dichotomische Einteilung in Adopter und Nicht-Adopter, das Verlassen der Prozessinnovation als vorrangig betrachteter Gegenstand (was zu einer eigenständigen Forschung im Marketing zur Diffusion neuer Produkte führte) sowie die dynamische Betrachtung von Determinanten und Variablen während des Diffusionsverlaufes selbst.

Diese zwei grundlegenden Stränge haben die empirische Forschung der siebziger und achtziger Jahre geprägt. So standen einerseits Faktoren zum ,Stand der Technik wie Komplexität, Teilbarkeit, Probierbarkeit und Kompatibilität mit bestehenden Strukturen im Vordergrund. Andererseits wurden die unterschiedlichen Verhaltensweisen der Unternehmen betrachtet. Dabei standen Faktoren wie Entscheidungsstrukturen, Strategie, involvierte Personen, Finanzierungsmöglichkeiten etc. im Zentrum der Analyse.

Diese Zweigleisigkeit der Ansätze schien auf Basis der klassischen Ökonomie unauflösbar. Erst die evolutionäre Ökonomie unter Berufung auf Schumpeter und unter Aufgabe der Gleichgewichtsannahmen und der Betonung der Dynamik als prägendes Element weisen einen neuen Weg. Dabei werden zentrale Begriffe der Biologie entlehnt: Selektion, Reproduktion und Mutation werden zur Beschreibung der Phänomene und Strukturen in der Wirtschaft verwendet. Allerdings sind Suchprozesse nicht nur zufällig, sondern können systematisch erfolgen. Dabei kann zwischen einer individualistisch-behavioristischen Schule und einer institutionell-historischen Richtung unterschieden werden. ${ }^{122}$ Die erste erklärt die Dynamik aufgrund der Eigenschaften der beteiligten Individuen oder Verhaltensunterschiede von Organisationen, während die zweite Richtung das Verhalten von Systemen und das Zusammenspiel von Akteuren und ihrer Umgebung betrachtet. 
Die mikro-ökonomische Fundierung durch Dosi war zentral: Lernprozesse vollziehen sich auf Basis des technologischen Wissens und der jeweiligen sozioökonomischen Randbedingungen. Dosi spricht hier von Paradigmen, die durchaus parallel bestehende Entwicklungsbahnen für Techniken und ihre Anwendung begründen. Dadurch werden parallel existierende best practices möglich, die später durch Selektionsprozesse zu dominanten Designs führen können. Dieses wurde dann um Betrachtungen zu Lock-in-Phänomenen und Pfadabhängigkeiten ergänzt.

Lundvall betrachtete im selben Jahr die Akteursgeflechte in unterschiedlichen Ländern und identifizierte "Innovationssysteme“, die die Technikadoption, aber auch Forschung und Entwicklung prägen. Dabei werden sowohl Akteursgruppen als auch ihre Beziehungen betrachtet und zur Erklärung der Vielfalt (diversity) hinzugezogen. Später wurde dieser Ansatz verfeinert hin zu regionalen und technologischen Innovationssystem-Konzepten.

Beide Ansätze führten zu einer Fülle empirischer Untersuchungen zu historischinstitutionellen Betrachtungen der Technikentwicklung sowie zu Verfeinerungen und Anwendungen in der Betriebswirtschaftslehre. Letztlich bilden sie eine Brücke zur sozial-, kulturwissenschaftlichen und zur historischen Forschung, bei der Erkenntnisse zu Anwendungskontexten als ebenso wichtig zu bewerten sind wie die Vorteilhaftigkeit und der wirtschaftliche Vorteil der Neuerung selbst.

Inwieweit Archäologie und Altertumsforschung diese Ansätze zur Erklärung verwenden können oder schon verwenden, bleibt zu diskutieren. Die Darstellung der wirtschaftswissenschaftlichen Diffusions- und Innovationsforschung erlaubt möglicherweise die Erinnerung an einen Kriterienkatalog, der es gestattet, schrittweise über den Ausschluss einzelner Determinanten ein klareres Bild zu gewinnen. So könnte an folgende Fragen erinnert werden:

- Wo liegen die Einsatzvoraussetzungen (physikalisch, geographisch etc.) für eine Neuerung überhaupt vor?

- Welche Verbesserung ist durch die Neuerung zu erreichen (Ertrag, Profitabilität, Zeitersparnis etc.)?

- Stellt die Innovation eine neue Lösung dar oder wird eine vorhandene substituiert?

- Welcher Aufwand und welches Risiko sind mit der Einführung verbunden?

- Ist die Einführung reversibel (Probierbarkeit)?

- Wie schnell erfolgt die Rückmeldung über den Erfolg der Neuerung an den Anwender selbst und an die bisherigen Nicht-Anwender und die potentiellen Adopter?

- Welche Wissensbestandteile sind in welcher Relation untereinander zur Einführung notwendig (Voraussetzungen und Kompetenzen bei den potentiellen Anwendern, ,Reisefähigkeit‘ kodifizierten Wissens, Schützbarkeit des Wissens, Anteil personengebundenen Erfahrungswissens)?

- Welches sind die Kommunikationskanäle für das Wissen über die Neuerung, ihren möglichen Erfolg sowie Erfahrungen anderer mit ihr (zufällige oder systematische Suchstrategien [Routinen])? 
- Welches soziokulturelle Umfeld (Innovationssystem inkl. Akteure, Arenen und Interaktionsmuster, Wettbewerbsintensität, (ggf. vermachtete) logistische oder geographische Engstellen) liegt vor und wie beeinflusst es den Umgang mit Neuerungen?

- Gibt es parallele Entwicklungen (different best practices) und abweichende Entwicklungspfade mit Ko-Existenz von Lösungen über längere Zeiträume hinweg (Pfadabhängigkeit, Lock-Ins)?

Vielleicht können die neuen naturwissenschaftlichen Methoden der Archäologie zusammen mit dem historischen Wissen zu den soziokulturellen Entwicklungen die Fragen erhellen. $\mathrm{Zu}$ wünschen wäre es, da dies ebenfalls zum Verständnis der Mechanismen der heutigen Zeit beitragen könnte, selbst wenn diese in der Internet-Ära im Zeitraffer ablaufen.

\section{Literaturverzeichnis}

Abernathy/Utterback 1978

W. J. Abernathy/J. M. Utterback, Patterns of Industrial Innovation. In: Technology Review, Band 80, Nummer 7, 40-47.

Abernathy/Utterback 1982

W. J. Abernathy/J. M. Utterback, Patterns of Industrial Innovation. In: M. L. Tushman/ W. L. Moore (Hrsg.), Readings in the Management of Innovation, Boston, o.S.

Archibugi/Lundvall 200I

A. Archibugi/B.-A. Lundvall (Hrsg.), The Globalizing Learning Economy, Oxford University, New York.

Arnold 2003

H. Arnold, Technology Shocks - Origins, Managerial Responses and Firm Performance, Heidelberg.

\section{Bass 1969}

F. M. Bass, A New Product Growth Model for Consumer Durables. In: Management Science, Nummer 5, 215-227.

\section{Batten 1982}

D. Batten, On the Dynamics of Industrial Revolution. In: Regional Science and Urban Economics, Nummer I2, $449 \mathrm{ff}$.

\section{Behrends 1996}

R.-H. Behrends (Hrsg.), Faustkeil, Urne, Schwert - Archäologie in der Region Karlsruhe, Badenia Verlag, Karlsruhe.

Beise 200I

M. Beise, Lead Markets: Country Specific Success Factors of the Global Diffusion of Innovations, Heidelberg. 


\section{Bewley/Fiebig 1988}

R. Bewley/D. G. Fiebig, A Flexible Logistic Growth Model with Publication in Telecommunications. In: International Journal of Forecasting, Band 4, Nummer 2, I77-I92.

\section{Bhargava 1989}

S. C. Bhargava, Generalized Lotka-Volterra Equations and the Mechanism of Technological Substitutions. In: Technological Forecasting and Social Change, Nummer 35 (I989), 3Igff.

\section{Blackman I97I}

A. W. Blackman, The Rate of Innovation in the Commercial Aircraft Jet Engine Market. In: Technological Forecasting and Social Change, Nummer 2, 269-276.

\section{Bollmann 1990}

P. Bollmann, Technischer Fortschritt und wirtschaftlicher Wandel: Eine Gegenüberstellung neoklassischer und evolutonischer Innovationsforschung, Heidelberg.

\section{Brown I981}

L. A. Brown, Innovation Diffusion - A New Perspective, London/New York.

\section{Bruckner et al. 1993}

D. Bruckner/F. Ebeling/V. Jimenez Montano/G. Scharnhorst, Technological Innovations - A Self Organisation Approach, Wissenschaftszentrum Berlin, FS II 93-302, April I993, Berlin.

\section{Burgelman 1984}

R. Burgelman, Managing the Internal Corporate Venturing Process. In: Sloan Management Review, Band 25, Nummer 2, 33-48.

\section{Burgelman et al. 2004}

R. Burgelman/C. Christensen/S. Wheelwright Strategic Management of Technology and Innovation, 4. Ausgabe, Boston et al.

\section{Cainarca et al. 1992}

G. C. Cainarca/M. G Colombo, Agreements between Firms and the Technological Life Cycle Model: Evidence from Information Technologies. In: Research Policy, Band 2I, Nummer I, $45^{-62 .}$

\section{Callon 1995}

M. Callon, Technological Conception and Adoption Network: Lessons for the CTA Practitioner. In: A. Rip/T. J. Misa/J. Schot (Hrsg.), Managing Technology in Society - The Approach of Constructive Technology Assessment, London.

\section{Christensen 1997}

C. R. Christensen, The Innovator's Dilemma - When New Technologies Cause Great Firms to Fail, Boston MA.

\section{Coombs/Hull 1998}

R. Coombs/R. Hull, Knowledge Management Practices and Path-Dependency in Innovation. In: Research Policy, Nummer 27, 237-253.

Cyert/March 1963

R. M. Cyert/J. G. March, A Behavorial Theory of the Firm, Englewood/Cliffs N.J. 
Dalum et al. 2002

B. Dalum/Ch. R. Pedersen/G. Vilumsen, Technological Life Cycles: Regional Clusters Facing Disruption, DRUID Working Paper, Nummer O2-IO, I-23.

Davies 1979

S. Davies, The Diffusion of Process Innovations, Cambridge.

\section{Dixon I980}

R. Dixon, Hybrid Corn Revisited. In: Econometrica, Nummer 48, I45I-I46I (September).

\section{Dodgson/Rothwell I994}

M. Dodgson/R. Rothwell (Hrsg.), The Handbook of Industrial Innovations, Aldershot/ Brookfield.

\section{Dosi 1982}

G. Dosi, Technological Paradigms and Technological Trajectories. In: Research Policy, Band II, Nummer 3 .

\section{Dosi I99I}

G. Dosi, The Research on Innovation Diffusion, An Assessment. In: N. Nakicenovic/ A. Grübler (Hrsg.), Diffusion of Technologies and Social Behaviour, Berlin u.a.

\section{Dosi 1998}

G. Dosi, Sources, Procedures and Microeconomics Effects of Innovation. In: Journal of Economic Literature, Band XXVI, II20-II7I.

\section{Dosi et al 1988}

G. Dosi/ L. Orsenigo/ G. Silverberg, Technical Change and Economic Theory, London.

\section{Dreher 1997}

C. Dreher, Technologiepolitik und Technikdiffusion - Auswahl und Einsatz von Förderinstrumenten am Beispiel der Fertigungstechnik, Karlsruher Beiträge zur wirtschaftspolitischen Forschung - Schriftenreihe des Instituts für Wirtschaftspolitik und Wirtschaftsforschung IWW. In: R. Funck/W. Rothengatter (Hrsg.), Band 5; Nomos-Verlag, Baden-Baden, I997 (zugleich als Dissertation: Dreher, Carsten: Der Beitrag der Diffusionsforschung zur Auswahl geeigneter technologiepolitischer Instrumente, Fakultät für Wirtschaftswissenschaften, Universität Karlsruhe I996).

\section{Dreher 2008}

C. Dreher, Herausforderungen des ganzheitlichen Innovationsverständnisses an das deutsche Innovationssystem - Stärken, Defizite, offene Fragen und Chancen der Partizipation auf dem Weg zu einer modernen Innovationspolitik, Expertise für die Hans-Böckler-Stiftung, Düsseldorf.

\section{Duchesneau et al. 1979}

T. D. Duchesneau/S. F. Cohn/J. E. Dutton, A Study of Innovation in Manufacturing: Determinants, Processes, and Methodological Issues, Band 2: Case Studies of Innovation Decision Making in the U.S. Footwear Industry, Toronto.

\section{Easingwood et al I98I}

C. J.Easingwood/V. Mahajan/E. Muller, A Nonsymmetric Responding Logistic Model for Technological Substitution. In: Technological Forecasting and Social Change, Nummer 20, I99-2I3 (Oktober). 


\section{Easingwood et al 1983}

C. J.Easingwood/V. Mahajan/E. Muller, A Nonuniform Influence Innovation Diffusion Model of New Product Acceptance. In: Marketing Science, Nummer 2, 273-296 (Summer).

\section{Edquist 1997}

C. Edquist, Systems of Innovation: Technologies, Institutions and Organizations, London/Washington.

\section{Erdmann 1993}

G. Erdmann, Elemente einer evolutionären Innovationstheorie, Tübingen.

\section{Etzkowitz/Leyendorff 2000}

H. Etzkowitz/L. Leyendorff, The Dynamics of Innovation: From National Systems and Mode to a Triple Helix of University - Industry - Government Relations. In: Research Policy, Nummer 29, I09-I23.

\section{Ewers et al. 1990}

H. J. Ewers/C. Becker/M. Fritsch, Wirkungen des Einsatzes computergestützter Techniken in Industriebetrieben. In der Reihe: Arbeitsmarktwirkungen moderner Technologien, Band 6, Berlin/New York, I990.

\section{Fisher/Pry I97I}

J. C. Fisher/R. H. Pry, A Simple Substitution Model of Technological Change. In: Forecasting and Social Change, Nummer 3, 75-88.

\section{Floyd 1962}

R. W. Floyd, Algorithm 97: Shortest Path. In: Communications of the ACM, Band 6, Nummer 5, 344-348.

\section{Francis/Bessant 2005}

D. Francis/J. Bessant, Targeting Innovation and Implications for Capability Development. In: Technovation, Nummer 25, I7I-I83.

\section{Freeman 1982}

Ch. Freeman, The Economics of Industrial Innovation, London.

\section{Freeman 1987}

Ch. Freeman, Technical Change and Full Employment, Oxford et al.

\section{Freeman/Soete 1997}

Ch. Freeman/L. Soete, The Economics of Industrial Innovation (Third Edition), Cambridge.

\section{Frenzel/Grupp 2009}

A. Frenzel/H. Grupp, Using Models of Innovation to Forecast Market Success: a Practitioners' Guide. In: Research Evaluation I8(I), March 2009, 39-50.

\section{Geroski 2000}

P. A. Geroski, Models of Technology Diffusion. In: Research Policy, Nummer 29, 603-625.

\section{Gerybadze 2004}

A. Gerybadze, Technologie- und Innovationsmanagement, München. 
Gibbons et al. 1994

M. Gibbons/C. Limoges/H. Nowotny/S. Schwartzmann/P. Scott/M. Trow, The New Production of Knowledge, The Dynamics of Science and Research in Contemporary Societies, London u.a.

\section{Gierl 1987}

H. Gierl, Die Erklärung der Diffusion technischer Produkte, Berlin.

\section{Gold I98I}

B. Gold, Technological Diffusion in Industry: Research Needs and Shortcomings. In: Journal of Industrial Economics, Band 29, Nummer 3, 247-269.

\section{Gold 1983}

B. Gold, On the Adaption of Technological Innovations in Industry: Superficial Models and Complex Desicion Processes. In: M. McDonald/D. Lamberton/Th. Mandevolle (Hrsg.), The Trouble with Technology - Explorations in the Process of Technological Change, London, IO4-I2I.

\section{Gottinger 1986}

H.-W. Gottinger, Adoption Decisions and Diffusion of Innovations: Part I. In: International Journal of Technology Management, Nummer I, 439-455.

\section{Grübler 1992}

A. Grübler, Introduction to Diffusion Theory. In: R. U. Ayres/W. Hyewood/ I. Tchijov (Hrsg.), Computer-Integrated Manufacturing Vol. III: Models, Case Studies and Forecasts of Diffusion, London, 3-52.

\section{Grupp 1997}

H. Grupp, Messung und Erklärung des technischen Wandels, Heidelberg.

\section{Grupp et al. 2004}

H. Grupp/H. Legler/G. Licht, Technologie und Qualifikation für neue Märkte. Ergänzender Bericht zur technologischen Leistungsfähigkeit Deutschlands 2003-2009, BMBF, Berlin/ Bonn.

\section{Habermeier 1989}

K. F. Habermeier, Competing Technologies, the Leaning Curve and Rational Expectations. In: European Economic Review, Nummer 33, I293-I3II.

\section{Hauschildt 2004}

J. Hauschildt, Innovationsmanagement, München, 3. Aufl.

\section{Hendry 1972}

I. Hendry, The Three Parameter Approach to Long-range Forecasting. In: Long Range Planning, Nummer 51, 40-45 (March).

\section{Herstatt/Verworn 2003}

C. Herstatt/B. Verworn (Hrsg.), Management der früheren Innovationsphasen - Grundlagen, Methoden, Neue Ansätze, Wiesbaden.

Heuss 1965

E. Heuss, Allgemeine Markttheorie, Tübingen. 


\section{Hsia 1973}

R. Hsia, Technological Change in the Industrial Growth of Hong Kong. In: B. R. Williams (Hrsg.): Science and Technology in Economic Growth, Macmillan, New York, N.Y.

\section{Jeuland I98I}

A. P. Jeuland, Parsimonious Models of Diffusion of Innovation: Part. A.. In: Derivations and Comparisons, Working Paper, Graduate School of Business, University of Chicago, IL, USA.

\section{Kashenas/Stoneman 1995}

M. Kashenas/P. Stoneman, Technological Diffusion. In: P. Stoneman (Hrsg.), Handbook of the Economics of Innovation and Technological Change, 265-297.

\section{Kleine 1983}

J. Kleine, Investitionsverhalten bei Prozessinnovationen - Ein Beitrag zur mikroökonomischen Diffusionsforschung, Frankfurt.

\section{Klepper 1996}

S. Klepper, Entry, Exit, Growth, and Innovation over the Product Life Cycle. In: American Economic Review, Band 86, Nummer 3, 562-583.

\section{Kline/Rosenberg 1986}

S. J. Kline./N. Rosenberg An Overview on Innovation. In: R. Landau/N. Rosenberg (Hrsg): The Positive Sum Strategy, Washington DC, 275-305.

\section{Klophaus 1995}

R. Klophaus, Anwendungsprobleme bei der Diffusionsprognose und -steuerung: Das Ende der klassischen Diffusionsmodellierung?. In: Marketing ZFP, Heft 2, II. Quartal I995, 89-98.

\section{Kortmann 1995}

W. Kortmann, Diffusion, Marktentwicklung und Wettbewerb - Eine Untersuchung über die Bestimmungsgründe zu Beginn des Ausbreitungsprozesses technologischer Produkte, Frankfurt a.M.

\section{Lissoni/Metcalfe 1994}

F. Lissoni/J. S. Metcalfe, Diffusion of Innovation Ancient and Modern: A Review of the Main Themes. In: M. Dodgson/R. Rothwell (Hrsg.), The Handbook of Industrial Innovations, Aldershot, Brookfield, I06-I4I.

\section{Lundvall 1992}

B.-A. Lundvall (Hrsg.), National Systems of Innovation: An Analytical Framework, London.

\section{Lundvall et al 2002}

B.-A. Lundvall/B. Johnson/E. S. Andersen/B. Dalum, National Systems of Production, Innovation and Competence Building. In: Research Policy, Nummer 3I, 213-23.

\section{Maas 1990}

Ch. Maas, Determinanten betrieblichen Innovationsverhaltens - Theorie und Expertise, Berlin.

\section{Mahajan et al. I99I}

V. Mahajan/E. Muller/F. M. Bass, New Product Diffusion Models in Marketing - A Review and Directions for Research. In: N. Nakicenovic/A. Grübler (Hrsg.), Diffusion of Technologies and Social Behavior, Berlin u.a., I25-I77. 
Malerba 2002

F. Malerba, Sectoral Systems of Innovation and Production. In: Research Policy, Nummer 3I, $247-264$.

Malerba 2004

F. Malerba (Hrsg.), Sectoral Systems of Innovation: Concepts, Issues and Analysis of Six Major Sectors in Europe, Cambridge.

\section{Mansfield i96I}

E. Mansfield, Technical Change and the Rate of Imitation. In: Econometrica, Band 29, Nummer $4,74 \mathrm{I}-766$.

Mansfield i968a

E. Mansfield, Industrial Research and Technological Innovation - An Econometric Analysis, New York.

\section{Mansfield i968b}

E. Mansfield, The Economics of Technological Change, New York, NY.

\section{Mansfield 1977}

E. Mansfield, The Production Application of New Industrial Technology, New York.

\section{Mansfield 1984}

E. Mansfield, Economic Effects of Research and Development, the Diffusion Process, and Public Policy. In: R. A. Goodmann/J. Pavón (Hrsg.), Planning for National Technology Policy, Praeger, NY, N.Y.

\section{Marchetti/Nakicenovic 1979}

C. Marchetti/N. Nakicenovic, The Dynamics of Energy Systems and the Logistic Substitution Model, RR-79-I3, IIASA, Laxenburg, Austria.

\section{Mauri/McMillan 1999}

A. J. Mauri/G. S. McMillan, The Influence of Technology on strategic Alliances: An Application of the Utterback and Abernathy Model of Product and Process Innovation. In: International Journal of Innovation Management, Band 3, Nummer 4, 367-378.

\section{McGowan 1986}

I. McGowan, The Use of Growth Curves in Forecasting Market Development. In: Journal of Forecasting, Band 5, Nummer I, 69-7I.

\section{Metcalfe 1983}

J. S. Metcalfe, Impulse and Diffusion in the Study of Technical Change. In: Ch. Freeman (Hrsg.), Long Waves in the World Economy. Butterworths, London, UK, S. IO2-II4.

\section{Metcalfe 1995}

J. S. Metcalfe, The Economic Foundations of Technology Policy: Equilibrium and Evolutionary Perspectives. In: P. Stoneman (Hrsg.): Handbook of Economics of Innovation and Technology Change, Oxford. 


\section{Meyer-Krahmer 200I}

F. Meyer-Krahmer, The German Innovation System. In: P. Laredo/P. Mustar (Hrsg.), Research and Innovation Policies in the New Global Economy: An International Comparative Analysis, Cheltenham, 205-252.

\section{Meyer-Krahmer 2004}

F. Meyer-Krahmer, Innovations- und Technologiepolitik. In: F. Gerlach/A. Ziegler (Hrsg.), Neuere Herausforderungen der Strukturpolitik, I8I-204.

\section{Meyer-Krahmer/Dreher 2004}

F. Meyer-Krahmer/C. Dreher, Neuere Betrachtungen der Technikzyklen und Implikationen auf die Fraunhofer Gesellschaft. In: D. Spath (Hrsg): Forschungs- und Technologiemanagement, München.

\section{Nakicenovic 1984}

N. Nakicenovic, Growth to Limits, Long Waves and the Dynamics of Technology, Dissertation, Sozial- und Wirtschaftswissenschaftliche Fakultät, Universität Wien.

\section{Nelder 1962}

J. A. Nelder, An Alternative Form of a generalized Logistic Equation. In: Biometrics, Nummer I8, GI4-6i6.

\section{Nelson/Winter 1982}

R. R. Nelson/S. Winter, Government and Technical Progress: A Cross-Industry Analysis, New York u.a.

\section{Norton/Bass 1987}

J. Norton/F. Bass, A Diffusion - Theory Model of Adoption and Substitution for Successive Generations of High Technology Products. In: Management Science, Nummer 33, 1069-1086.

\section{OECD (Hrsg.) 1997}

The Oslo-Manual - The Proposed Guidelines for Collecting and Interpreting Technological Innovation Data, Paris.

\section{Pavitt 1984}

K. Pavitt, Sectoral Patterns of Technical Change - Towards a Taxanomy and a Theory. In: Research Policy, Nummer 13, 77-99.

\section{Porter 1990}

M. Porter, The Competitive Advantage of Nations, New York.

\section{Ray 1989}

G.F. Ray, Full Circle: The Diffusion of Technology. In: Research Policy, No. I8, I-I8.

\section{Reger/Schmoch 1996}

G. Reger/U. Schmoch, (Hrsg.), Organisation of Science and Technology at the Watershed, The Academic and Industrial Perspective. In: Series of the Fraunhofer Institute for Systems and Innovation Research (ISI), Band 3, Heidelberg. 
Renn et al. 2009

J. Renn/M. Hyman/D. Potts, Die Wege des Wissens in der alten Welt - Ein kurzer Überblick, Essay. In: Topoi Raumwissen I. Jg. I-2009, S. 64-69.

Richards 1959

F. J. Richards, A Flexible Growth Function for Empirical Use. In: Journal of Experimental Botany, Number I0, 290-300.

\section{Rickerby/Matthews I99I}

D. S. Rickerby/A. Matthews, Advanced Surface Coatings: A Handbook of Surface Engineering Blackie Glasgow and London, New York, 343-364.

\section{Rogers 1983}

E. M. Rogers, Diffusion of Innovation 3rd Edition, New York (1. Auflage I962, New York).

\section{Romeo 1977}

A. Romeo, The Rate of Imitation of a Capital-embodied Process Innovation. In: Economica, Nummer 44(173), 63-69.

\section{Rosenberg 1976}

N. Rosenberg, Perspectives on Technology, Cambridge, U.K.

\section{Rosenberg 1982}

N. Rosenberg, Inside the Black Box, Cambridge, U.K.

\section{Rothwell 1992}

R. Rothwell, Successful Industrial Innovation: Critical Factors for the I990's. In: R\&D Management, Band 22, Nummer 3, 22I-239.

\section{Russel I980}

T. Russel, Comments on „The Relationship between Diffusion Curves, Experience Curves, and Demand Elasticities for Consumer Durable Technological Innovations (Bass, I980)“. In: Journal of Business, Nummer 53, 69-73.

Schmoch et al. 2000

U. Schmoch/G. Licht/M. Reinhard (Hrsg.), Wissens- und Technologietransfer in Deutschland, Stuttgart.

\section{Schumpeter I9II}

J. A. Schumpeter, Theorie der wirtschaftlichen Entwicklung, München/ Leipzig, zitiert nach 6. Auflage, Berlin, I984.

\section{Schumpeter 1942}

J. A. Schumpeter, Capitalism, Socialism and Democracy; New York zitiert nach deutscher Übersetzung: Kapitalismus, Sozialismus und Demokratie, 4. Auflage, München, I975.

\section{Schwitalla 1993}

B. Schwitalla, Messung und Erklärung industrieller Innovationsaktivitäten mit einer empirischen Analyse für die westdeutsche Industrie, Heidelberg.

\section{Sharif/Kabir 1976}

M. N. Sharif/C. Kabir, A Generalized Model for Forecasting Technological Substitution. In: Technological Forecasting and Social Change, Nummer 8, 353-364. 


\section{Simon 1959}

H. A. Simon, Theories of Decision Making in Economics. In: American Economic Review, Nummer 49, 253-283.

\section{Simon 1975}

P. Simon, Models of Process Diffusion and Entry in the US Chemical Industry, Ph.D. Dissertation, University of Pennsylvania, Philadelphia, PA.

\section{Skiadas 1986}

C. Skiadas, Innovation Diffusion Models Expressing Asymmetry and/or Positively or Negatively Influencing Factors. In: Technological Forecasting and Social Change, Nummer 30, 313-330.

\section{Stoneman 1983}

P. Stoneman, The Economic Analysis of Technological Change, Oxford.

\section{Stoneman 1987}

P. Stoneman, Some Analytical Observations on Diffusion Policies. In: P. Dasgupta/P. Stoneman (Hrsg.), Economic Policy and Technological Performance, Cambridge, I54-I68.

\section{Stumpfe}

J. Stumpfe, Product Design and Manufacturing Process: Dynamic Implications for Innovation Management. Arbeitspapier Industrieseminar der Universität Mannheim.

\section{Teece 2003}

D. J. Teece, Essays in Technology Management and Policy: Selected Papers of David Teece, New Jersey.

\section{Teotia/Raju 1986}

A. P. S. Teoti/P. S. Raju, Forecasting the Market Penetration of New Technologies Using a Combination of Economic Cost and Diffusion Models. In: Journal of Product Innovation Management, Nummer 3, 225-237 (Dezember).

\section{Thiel I988}

W. Thiel, Mikroökonomische Fundierung des dynamischen Innovationswettbewerbs, Dissertation, Karlsruhe.

\section{Thirtle/Ruttan 1987}

C. G. Thirtle/V. W. Ruttan, The Role of Demand and Supply in the Generation and Diffusion of Technological Change. In: J. Lesourne/H. Sonnenschein (Hrsg.), Fundamentals of Pure and Applied Economics, Band 2I, Chur, London, Paris, New York.

Tidd et al 200I

J. Tidd/J. Bessant/K. Pavitt, Managing Innovation - Integrated Technological Market and Organisational Change, zweite Auflage, Chichester.

\section{Tijssen 2002}

R. J. W. Tijssen, Science Dependence of Technologies: Evidence from Intentions and Their Inventors. In: Research Policy, Band 31, 509-526.

\section{Utterback/Abernathy 1975}

J. M. Utterback/W. J. Abernathy, A Dynamic Model of Process and Product Innovation. In: The International Journal of Management Science, 3/6, 639-656. 
van de Ven et al. 1999

A. van de Ven/D. Polley/R. Garud/S. Venkataraman, The Innovation Journey, Oxford.

von Bertalanffy 1957

L. von Bertalanffy, Quantitative Laws in Metabolism and Growth. In: Quarterly Review of Biologie, Nummer 32, 2I7-23I.

von Hayek 1969

F. A. von Hayek, Freiburger Studien, gesammelte Werke, Tübingen.

von Hippel 1988

E. von Hippel, The Sources of Innovation, Oxford.

\section{Wengenroth 200I}

U. Wengenroth, Vom Innovationssystem zur Innovationskultur - Perspektivwechsel in der Innovationsforschung. In: J. Abele/G. Barkleit/T. Hänseroth (Hrsg.), Innovationskultur und Fortschrittserwartungen im geteilten Deutschland, Köln, 23-32.

Werker 2003

C. Werker, Innovation, Market Performance, and Competition: Lessons from a Product Life Cycle Model. In: Technovation, Nummer 23, 28I-290.

Weule 2002

$\mathrm{H}$. Weule, Integriertes Forschungs- und Entwicklungsmanagement, München.

\section{Witt 1987}

U. Witt, Individualistische Grundlagen der evolutorischen Ökonomik, Tübingen. 
